\title{
FINITE ELEMENT ANALYSIS OF CAUCHY-BORN APPROXIMATIONS TO ATOMISTIC MODELS
}

\author{
CHARALAMBOS MAKRIDAKIS AND ENDRE SÜLI
}

\begin{abstract}
This paper is devoted to a new finite element consistency analysis of Cauchy-Born approximations to atomistic models of crystalline materials in two and three space dimensions. Through this approach new "atomistic Cauchy-Born" models are introduced and analyzed. These intermediate models can be seen as first level atomistic/quasicontinuum approximations in the sense that they involve only short-range interactions. The analysis and the models developed herein are expected to be useful in the design of coupled atomistic/continuum methods in more than one dimension. Taking full advantage of the symmetries of the atomistic lattice we show that the consistency error of the models considered both in energies and in dual $W^{1, p}$ type norms is $\mathcal{O}\left(\varepsilon^{2}\right)$, where $\varepsilon$ denotes the interatomic distance in the lattice.
\end{abstract}

\section{INTRODUCTION}

Modern multiscale methods for the simulation of materials introduce several coupling mechanisms of the atomistic and the continuum descriptions aiming at the design of methods of "atomistic" accuracy with "continuum" cost. To understand these mechanisms and the behavior of the coupled models is a challenge both from the modeling point of view and from the computational perspective. It is known, for example, that ad-hoc coupling of models may lead to undesirable computational artifacts $[16,28]$. The development of the mathematical foundations of coupled multiscale models therefore seems necessary. Although the mathematical theory of multiscale models is still quite limited at present, it is hoped that, ultimately, the availability of a comprehensive mathematical theory of multiscale models will enhance the development of efficient, accurate and robust numerical algorithms for multiscale models. Indeed, the area of multiscale simulations in materials science is a very active field; see, for example, the review articles [29, 10]. In particular, a problem that has received considerable attention from the engineering as well as from the mathematical point of view is the atomistic-to-continuum passage (cf. [25, 9, 26, 2, 24, 32, 21]), and the corresponding coupled methods for crystalline materials (cf. [39, 7, 23, 10, 8, 4, 3, 5, 6, 15, 14, 36, 17, 16, $27,30,18,19,20,35,28,37,38,43,44,1,22,34,35,42,41])$.

Much of the literature on atomistic/continuum coupling in crystals is concerned with the "quasicontinuum" method [39] and its variants. In these methods, in regions of interest in the material (strong deformations, defects) the atomistic model is kept, while in regions of smooth deformations the atomistic model is replaced with a continuum model discretized by finite elements. Despite the increasing number of papers concerned with the numerical analysis of these methods, satisfactory analytical results are available in one space dimension only; in two and three space dimensions the precise formulation of efficient coupling methods is still in its infancy. 
This paper is devoted to a novel finite element consistency analysis of Cauchy-Born approximations to atomistic models of crystalline materials in two and three space dimensions. Through this approach new "atomistic Cauchy-Born" models are introduced and analyzed. These intermediate models can be seen as first level atomistic/quasicontinuum approximations in the sense that they involve only short-range interactions. The analysis and the models developed herein are expected to be useful in the design of coupled atomistic/continuum methods in several space dimensions. Specifically, we concentrate on the comparison of an atomistic model and its continuum Cauchy-Born approximation, as in [9, 24]; however, in contrast with the "finite-difference"-style analysis in [9, 24], here we develop a theoretical framework in the spirit of finite-element methods. Taking full advantage of the symmetries we show that the consistency error of the models considered in dual $W^{1, p}$ type norms is $\mathcal{O}\left(\varepsilon^{2}\right), \varepsilon$ being the interatomic distance. As a consequence we provide an alternative finite element proof of the second order consistency result of the continuum CauchyBorn model derived by E \& Ming [24] using finite difference techniques. In addition we derive consistency results for the energies, by showing that all models considered have energies that are $\mathcal{O}\left(\varepsilon^{2}\right)$ close to each other. The first consistency results of the energies for the continuum Cauchy-Born model approximating the atomistic model were derived in Blanc, LeBris \& Lions [9]. Our approach is based on the introduction and analysis of intermediate "atomistic Cauchy-Born" models. Since, most of the recent results concerning the construction and analysis of various quasicontinuum methods in one space dimension were based on the properties and the proper comparison of a similar intermediate atomistic Cauchy-Born model involving only short-range interactions we expect that our approach can provide the appropriate analytical framework for the construction and analysis of various atomisitic/continuum methods in multiple space dimensions.

1.1. Notation. Lattice, discrete domain, continuum domain. We consider a simple $d$-dimensional lattice, which is generated by $d$ linearly independent vectors of $\mathbb{R}^{d}, d=2,3$. For simplicity of the exposition we assume that the lattice $\mathfrak{L}_{\text {entire }}$ is generated by the unit coordinate vectors $e_{1}, \ldots, e_{d}$ of $\mathbb{R}^{d}$. The extension of the consistency analysis developed in this paper to include any $d$ linearly independent vectors of $\mathbb{R}^{d}$ is straightforward, since the general case can be obtained by applying an affine map. We note however that, unlike consistency, stability can be sensitive to the specific lattice structure (e.g. rectangular or triangular); see, [24], for example. We will consider discrete periodic functions on $\mathfrak{L}_{\text {entire }}$ defined over a "periodic domain' $\mathfrak{L}$. More precisely, let

$$
\begin{aligned}
\mathfrak{L}:=\left\{\ell=\left(\ell_{1}, \ldots, \ell_{d}\right)=\right. & n_{1} e_{1}+\cdots+n_{d} e_{d}: \\
& \left.\left(n_{1}, \ldots, n_{d}\right) \in \mathbb{Z}^{d} \cap\left[-N_{1}-1, N_{1}\right] \times \cdots \times\left[-N_{d}-1, N_{d}\right]\right\} .
\end{aligned}
$$

The actual configuration of the atoms is thus a subset of $\mathbb{R}^{d}$, which we call discrete domain and denote by $\Omega_{\mathrm{discr}}$; the corresponding continuum domain is denoted by $\Omega$; i.e.,

$$
\begin{aligned}
& \Omega_{\mathrm{discr}}:=\left\{x_{\ell}=\left(x_{\ell_{1}}, \ldots, x_{\ell_{d}}\right)=\varepsilon \ell, \quad \ell \in \mathfrak{L},\right\}, \\
& \Omega:=\left\{x \in\left[x_{-N_{1}-1}, x_{N_{1}}\right] \times \cdots \times\left[x_{-N_{d}-1}, x_{N_{d}}\right]\right\}^{o} .
\end{aligned}
$$

Here $\mathcal{O}^{\circ}$ denotes the interior of the set $\mathcal{O}^{\circ}$. Note that a possible confusion in the notation may arise when $\ell_{i}=i, i=1, \ldots, d$, since by $x_{i}$ we denote the coordinates of the continuum variable $x$; see below. It will be clear however from the analysis which is the continuum variable.

Functions and spaces. We consider atomistic deformations

$$
\begin{aligned}
& y_{\ell}=y\left(x_{\ell}\right), \quad \ell \in \mathfrak{L} \quad \text { of the form } \\
& y_{\ell}=\mathrm{F} x_{\ell}+v_{\ell}, \quad \text { with } v_{\ell}=v\left(x_{\ell}\right) \text { periodic with respect to } \mathfrak{L} .
\end{aligned}
$$


Here $\mathrm{F}$ is a constant $d \times d$ matrix with $\operatorname{det} \mathrm{F}>0$. The corresponding spaces for $y$ and $v$ are denoted by $\mathcal{X}$ and $\mathscr{V}$ and are defined as follows:

$$
\begin{aligned}
& \mathcal{X}:=\left\{y: \mathfrak{L} \rightarrow \mathbb{R}^{d}, \quad y_{\ell}=\mathrm{F} x_{\ell}+v_{\ell}, \quad v \in \mathscr{V}, \quad \ell \in \mathfrak{L}\right\}, \\
& \mathscr{V}:=\left\{u: \mathfrak{L} \rightarrow \mathbb{R}^{d}, \quad u_{\ell}=u\left(x_{\ell}\right) \quad \text { periodic with zero average with respect to } \mathfrak{L}\right\} .
\end{aligned}
$$

For functions $y, v: \mathfrak{L} \rightarrow \mathbb{R}^{d}$ we define the inner product

$$
\langle y, v\rangle_{\varepsilon}:=\varepsilon^{d} \sum_{\ell \in \mathfrak{L}} y_{\ell} \cdot v_{\ell}
$$

For a positive real number $s$ and $1 \leq p \leq \infty$ we denote by $W^{s, p}\left(\Omega, \mathbb{R}^{d}\right)$ the usual Sobolev space of functions $y: \Omega \rightarrow \mathbb{R}^{d}$; we shall use the same symbol for discrete functions defined on the lattice and for continuum functions. It will be clear from the context which of the two is intended in a specific instance. Further by $W_{\#}^{s, p}\left(\Omega, \mathbb{R}^{d}\right)$ we denote the corresponding Sobolev space of periodic functions with basic period $\Omega$. By $\langle\cdot, \cdot\rangle$ we denote the standard $L^{2}(\Omega)$ inner product. The space corresponding to $\mathcal{X}$ in which the minimizers of the continuum problem are sought is

$$
\begin{aligned}
& X:=\left\{y: \Omega \rightarrow \mathbb{R}^{d}, \quad y(x)=\mathrm{F} x+v(x), \quad v \in V\right\}, \quad \text { where } \\
& V:=\left\{u: \Omega \rightarrow \mathbb{R}^{d}, \quad u \in W^{k, p}\left(\Omega, \mathbb{R}^{d}\right) \cap W_{\#}^{1, p}\left(\Omega, \mathbb{R}^{d}\right), \quad \int_{\Omega} u \mathrm{~d} x=0\right\} .
\end{aligned}
$$

Henceforth, for the sake of notational simplicity, we shall suppress the symbol $\mathbb{R}^{d}$ in our notation for Sobolev spaces, and will simply write $W^{s, p}(\Omega)$ and $W_{\#}^{s, p}(\Omega)$.

Difference quotients and derivatives. We will use the notation

$$
\bar{D}_{\eta} y_{\ell}:=\frac{y_{\ell+\eta}-y_{\ell}}{\varepsilon}, \quad \ell, \ell+\eta \in \mathfrak{L},
$$

for the difference quotient (discrete derivative) in the direction of the vector $\eta$. For functions defined on the continuum domain, the following notation is used

$$
\begin{aligned}
\partial_{\zeta_{i}} \phi(\zeta) & :=\frac{\partial \phi\left(\zeta_{1}, \ldots, \zeta_{d}\right)}{\partial \zeta_{i}}, \quad \zeta=\left(\zeta_{1}, \ldots, \zeta_{d}\right), \\
\nabla_{\zeta} \phi(\zeta) & :=\left\{\partial_{\zeta_{i}} \phi(\zeta)\right\}_{i}, \\
\partial_{\alpha} v(x) & :=\frac{\partial v(x)}{\partial x_{\alpha}}, \\
\nabla u(x) & :=\left\{\frac{\partial u^{i}(x)}{\partial x_{\alpha}}\right\}_{i \alpha} .
\end{aligned}
$$

To avoid confusion we distinguish between derivatives with respect to arguments, denoted by $\partial_{\zeta_{i}}$, which usually appear in composite functions, and derivatives with respect to the spatial variable $x_{i}$, denoted by the symbol $\partial_{i}$.

Atomistic and Cauchy-Born potential. We consider the atomistic potential

$$
\Phi^{a}(y):=\varepsilon^{d} \sum_{\ell \in \mathfrak{L}} \sum_{\eta \in R} \phi_{\eta}\left(\bar{D}_{\eta} y_{\ell}\right)
$$

where $R$ is a given finite set of interaction vectors. We allow the potential to vary with the type of bond, i.e., $\phi_{\eta}$ may vary with $\eta$. On the other hand we assume standard conditions on the potential away from zero (cf. [9]): $\phi_{\eta}$ are functions defined on $\mathbb{R}^{d} \backslash\{0\}$, which are smooth for any $\zeta,|\zeta|>\rho$. In fact we assume that there exist $C_{\rho, k}=C(\rho, k) \geq 0$, such that $\left|D_{\zeta}^{k} \phi_{\eta}(\zeta)\right| \leq C_{\rho, k}$, for $|\zeta|>\rho$, and a multi-index $k$ of length 
$|k| \leq 3$. Notice that we have not imposed any symmetry hypotheses on the potentials $\phi_{\eta}$. In order to explore the consistency of the Cauchy-Born approximation, we will consider sufficiently smooth diffeomorphisms $y$ on $\Omega$. The assumption that $y$ is $1-1$ is natural since it excludes interpenetration. In addition, it leads to the lower bound $\left|\bar{D}_{\eta} y_{\ell}\right| \geq \alpha(y, \eta)>0$, [9], which is required in the course of bounding derivatives of $\phi_{\eta}\left(\bar{D}_{\eta} y_{\ell}\right)$. It will be assumed throughout the paper that whenever the potential is applied to a smooth function $y, y$ is a diffeomorphism on the domain $\Omega$.

For a given field of external forces $f: \mathfrak{L} \rightarrow \mathbb{R}^{d}$, where $f_{\ell}=f\left(x_{\ell}\right)$, the atomistic problem reads as follows:

$$
\text { find a local minimizer } y^{a} \text { in } \mathcal{X} \text { of : }
$$

$$
\Phi^{a}(y)-\langle f, y\rangle_{\varepsilon} \text {. }
$$

If such a minimizer exists, then

$$
\left\langle D \Phi^{a}\left(y^{a}\right), v\right\rangle_{\varepsilon}=\langle f, v\rangle_{\varepsilon}, \quad \text { for all } v \in \mathscr{V},
$$

where

$$
\begin{aligned}
\left\langle D \Phi^{a}(y), v\right\rangle_{\varepsilon} & :=\varepsilon^{d} \sum_{\ell \in \mathfrak{L}} \sum_{\eta \in R} \sum_{i=1}^{d} \partial_{\zeta_{i}} \phi_{\eta}\left(\bar{D}_{\eta} y_{\ell}\right)\left[\bar{D}_{\eta} v_{\ell}\right]_{i} \\
& =\varepsilon^{d} \sum_{\ell \in \mathfrak{L}} \sum_{\eta \in R} \nabla_{\zeta} \phi_{\eta}\left(\bar{D}_{\eta} y_{\ell}\right) \cdot \bar{D}_{\eta} v_{\ell} .
\end{aligned}
$$

Throughout the rest of the paper we shall use the summation convention for repeated indices.

The corresponding Cauchy-Born stored energy function is

$$
W(\mathrm{~F})=W_{C B}(\mathrm{~F}):=\sum_{\eta \in R} \phi_{\eta}(\mathrm{F} \eta) .
$$

Then, the continuum Cauchy-Born model is stated as follows:

$$
\text { find a local minimizer } y^{C B} \text { in } X \text { of : }
$$

$$
\Phi^{C B}(y)-\langle f, y\rangle,
$$

where the external forces $f$ are appropriately related to the discrete external forces and

$$
\Phi^{C B}(y):=\int_{\Omega} W_{C B}(\nabla y(x)) \mathrm{d} x .
$$

If such a minimizer exists, then

$$
\left\langle D \Phi^{C B}\left(y^{C B}\right), v\right\rangle=\langle f, v\rangle, \quad \text { for all } v \in V,
$$

where

$$
\left\langle D \Phi^{C B}(y), v\right\rangle=\int_{\Omega} S_{i \alpha}(\nabla y(x)) \frac{\partial v^{i}(x)}{\partial x_{\alpha}} \mathrm{d} x=\int_{\Omega} S_{i \alpha}(\nabla y(x)) \partial_{\alpha} v^{i}(x) \mathrm{d} x, \quad v \in V .
$$

Here the stress tensor $S$ is defined, as usual, by

$$
S:=\left\{\frac{\partial W(\mathrm{~F})}{\partial F_{i \alpha}}\right\}_{i \alpha} .
$$


A simple calculation yields the following relation between the stress tensor and the atomistic potential:

$$
\begin{aligned}
S_{i \alpha} & =\frac{\partial W(\mathrm{~F})}{\partial F_{i \alpha}}=\frac{\partial}{\partial F_{i \alpha}} \sum_{\eta \in R} \phi_{\eta}(\mathrm{F} \eta) \\
& =\frac{\partial}{\partial F_{i \alpha}} \sum_{\eta \in R} \phi_{\eta}\left(F_{j \beta} \eta_{\beta}\right)=\sum_{\eta \in R} \partial_{\zeta_{i}} \phi_{\eta}\left(F_{j \beta} \eta_{\beta}\right) \frac{\partial}{\partial F_{i \alpha}} F_{j \beta} \eta_{\beta} \\
& =\sum_{\eta \in R} \partial_{\zeta_{i}} \phi_{\eta}(\mathrm{F} \eta) \eta_{\alpha} .
\end{aligned}
$$

1.2. Main results. The question whether, and under what conditions, the continuum Cauchy-Born model (1.5) approximates (1.3) is very delicate. The analytical assessment of the quality of an approximating scheme in Numerical Analysis is based on the notions of consistency, stability and convergence. Consistency essentially refers to the extent to which an exact smooth solution fails to satisfy the numerical scheme. Given that the stability of the approximating scheme is satisfactory, usually the consistency error determines the order and therefore the quality of the approximation. It is to be noted that the consistency error depends in an essential manner on the norm we use to measure it. The choice of the norm is, in turn, dictated by the chosen method of stability/convergence analysis.

In this paper we focus on the consistency analysis of Cauchy-Born approximations. We then briefly discuss the consequences of our results in the convergence analysis. To be more precise, let us assume that $y$ is a smooth solution of the Cauchy-Born problem (1.6). Then, our goal is to quantify the size of

$$
\left\langle D \Phi^{a}(y), v\right\rangle_{\varepsilon}-\langle f, v\rangle_{\varepsilon}, \quad \text { for all } v \in \mathscr{V} \quad \text { such that }\|v\|_{W^{1, p}(\Omega)}=1 .
$$

Here by $\|v\|_{W^{1, p}(\Omega)}$ we denote the $W^{1, p}$ norm of the bilinear interpolant of $v \in \mathscr{V}$. In addition, $y$ is identified with its bilinear interpolant. Elements of $\mathscr{V}$ will be identified with elements of the finite element space $\boldsymbol{V}_{\varepsilon}$, see Section 2 for the precise definitions. Modulo a data approximation error in $f$, it suffices to estimate

$$
\mathcal{C}_{V}(y):=\sup \left\{\left|\left\langle D \Phi^{a}(y), v\right\rangle_{\varepsilon}-\left\langle D \Phi^{C B}(y), v\right\rangle\right|: \quad v \in \mathscr{V} \quad \text { with }\|v\|_{W^{1, p}(\Omega)}=1\right\},
$$

where in the last relation $y$ is any smooth function. We we shall refer to $\mathcal{C}_{V}(y)$ as the variational consistency error. Similarly, one can define the energy consistency error

$$
\mathcal{C}_{\mathcal{E}}(y):=\left|\Phi^{a}(y)-\Phi^{C B}(y)\right| .
$$

Our aim is to show that both consistency errors are of second order in the lattice spacing $\varepsilon$; cf. Sections 4 and 5. Our assumptions on the potentials and on $y$ are standard; see the discussion following equation (1.2). Related results for energy consistency and variational consistency in the case of a single potential $\phi_{\eta}=\phi$ were derived by Blanc, LeBris \& Lions [9] and by E \& Ming [24], respectively. In [9] boundary effects were taken into account.

We note that our second order consistency results do not require symmetry of the potentials. Thus, Theorem 4.2, where we show that $\mathcal{C}_{\mathcal{E}}(y)=\mathcal{O}\left(\varepsilon^{2}\right)$ can be seen as an extension of Theorem 1 in [9] where under similar assumptions on the potential it was proved that $\mathcal{C}_{\mathcal{E}}(y) \rightarrow 0$ as $\varepsilon \rightarrow 0$. The estimate $\mathcal{C}_{\mathcal{E}}(y)=\mathcal{O}\left(\varepsilon^{2}\right)$ for symmetric potentials was proved in Theorem 3 of [9] allowing infinite-range interactions. Regarding variational consistency, it was shown in [24] by asymptotic methods, still under the assumption of symmetric potentials, that $\left|\left\langle D \Phi^{a}(y), v\right\rangle_{\varepsilon}-\left\langle D \Phi^{C B}(y), v\right\rangle\right| \leq C(y, v) \varepsilon^{2}$, with $C(y, v)$ depending on higher order derivatives of $y$ and $v$.

Our main contribution in this paper is the analytical approach proposed, which is based on finite element analysis, and the new construction of an intermediate "atomistic Cauchy-Born" model. The analysis of [9] and [24] is based on finite difference techniques and on the direct comparison of the atomistic and 
continuum Cauchy-Born models. The proposed "atomistic Cauchy-Born" model, see Section 2.3, is not the standard model that one gets by replacing in the atomistic potential long-range interactions by short-range ones. As was mentioned before, the available results concerning the construction and analysis of various quasicontinuum methods in one space dimension were heavily based on the use of intermediate atomistic Cauchy-Born models. The definition of these intermediate models is obvious in one dimension but, as our analysis shows, this is not so in multiple space dimensions. The finite element approach taken in this paper is a natural choice for coupled methods, since, by construction, atomistic/continuum coupling methods assume finite element discretization of the continuum region. We therefore expect that our approach can provide the appropriate analytical framework for the construction and analysis of various atomisitic/continuum methods in multiple space dimensions, which is at present lacking.

The paper is organized as follows. Section 2 is devoted to the introduction of the finite element notation and the construction of the atomistic Cauchy-Born model starting from the continuum model and performing appropriate approximation steps. In Section 3 we show that the atomistic model approximates the atomistic Cauchy-Born model with the desired accuracy. The use of the mesh symmetries is important in the analysis. Section 4 provides our main results in the two-dimensional case regarding the order of accuracy of the energy and variational consistencies related to the comparison of the atomistic and Cauchy-Born continuum models. Section 5 is devoted to the extension of our results to three space dimensions. Finally, in Section 6 we briefly discuss the applicability of our results in the convergence analysis, under suitable stability hypotheses.

\section{Construction of an atomistic CAUChy-Born Model}

In the sequel we provide a link between the continuum model and the atomistic model by introducing an intermediate model, which we call atomistic Cauchy-Born model (A-CB). To derive this model, we start from the continuum model and perform appropriate approximation steps, which finally yield the A-CB. The final model has consistency error of the order $\mathcal{O}\left(\varepsilon^{2}\right)$ compared to the continuum Cauchy-Born model. In the next section we show that the A-CB has $\mathcal{O}\left(\varepsilon^{2}\right)$ consistency error compared to the original atomistic model.

Bilinear finite elements on the lattice. Let $\boldsymbol{V}_{\varepsilon}$ be the space of continuous piecewise bilinear periodic functions on the lattice $\mathfrak{L}$. More precisely, let

$$
\begin{aligned}
& \mathcal{T}:=\left\{K \subset \Omega: \quad K=\left(x_{\ell_{1}}, x_{\ell_{1}+1}\right) \times\left(x_{\ell_{2}}, x_{\ell_{2}+1}\right), \quad x_{\ell}=\left(x_{\ell_{1}}, x_{\ell_{2}}\right) \in \Omega_{\mathrm{discr}}\right\}, \\
& \boldsymbol{V}_{\varepsilon}:=\left\{v: \bar{\Omega} \rightarrow \mathbb{R}^{2}, \quad v \in C(\bar{\Omega}),\left.v\right|_{K} \in \mathbb{Q}_{1}(K) \text { and } v_{\ell}=v\left(x_{\ell}\right) \quad \text { periodic with respect to } \mathfrak{L}\right\},
\end{aligned}
$$

where $\mathbb{Q}_{1}(K)$ denotes the set of all bilinear functions on $K$; i.e., $\left.v\right|_{K}(x)=\alpha_{0}+\alpha_{1} x_{1}+\alpha_{2} x_{2}+\alpha_{3} x_{1} x_{2}$. It is then well known that the elements of the linear space $\boldsymbol{V}_{\varepsilon}$ can be expressed in terms of the nodal basis functions, $\Psi_{\ell}=\Psi_{\ell}(x), \ell \in \mathfrak{L}$, as

$$
v(x)=\sum_{\ell \in \mathfrak{L}} v_{\ell} \Psi_{\ell}(x)=\sum_{\ell \in \mathfrak{L}} v_{\ell} \Psi_{\ell_{1}}\left(x_{1}\right) \Psi_{\ell_{2}}\left(x_{2}\right), \quad v_{\ell}=v\left(x_{\ell}\right),
$$

where we have used the fact that $\Psi_{\ell}(x)$ can be written as the tensor product of the standard one-dimensional piecewise linear hat functions $x_{1} \mapsto \Psi_{\ell_{1}}\left(x_{1}\right)$ and $x_{2} \mapsto \Psi_{\ell_{2}}\left(x_{2}\right)$ with respect to the $x_{1}$ and $x_{2}$ variable, respectively. Here $\Psi_{\ell_{1}}\left(x_{\tilde{\ell}_{1}}\right)=\delta_{\ell_{1} \tilde{\ell}_{1}}$ and $\Psi_{\ell_{2}}\left(x_{\tilde{\ell}_{2}}\right)=\delta_{\ell_{2} \tilde{\ell}_{2}}$.

2.1. Preliminaries. We shall make frequent use of the following version of the Bramble-Hilbert lemma [13, Theorem 4.1.3]. Its proof is completely straightforward and is therefore omitted. 
Lemma 2.1. Let $\mathcal{O}$ be a bounded open set in $\mathbb{R}^{d}$ and suppose that $1 \leq p \leq \infty$ and $s \geq 0$. Suppose further that $\zeta$ is a linear functional on a linear subspace $\mathbb{H}$ of $W^{s, p}(\mathcal{O})$ with the following property:

$$
\exists C_{0}>0 \quad \forall v \in \mathbb{H}:|\zeta(w)| \leq C_{0}\|w\|_{W^{s, p}(\mathcal{O})} .
$$

Then, for any $w \in \mathbb{H}$ and any set $\mathbb{S} \subset \operatorname{Ker}(\zeta)$ we have that $|\zeta(w)| \leq C_{0} \inf _{\varphi \in \mathbb{S}}\|w-\varphi\|_{W^{s, p}(\mathcal{O})}$.

If, in addition, there exists a positive constant $C_{1}$, independent of $\operatorname{diam}(\mathcal{O})$, and a real number $t>s$ such that

$$
\inf _{\varphi \in \mathbb{S}}\|w-\varphi\|_{W^{s, p}(\mathcal{O})} \leq C_{1}(\operatorname{diam}(\mathcal{O}))^{t-s}[w]_{W^{t, p}(\mathcal{O})} \quad \forall w \in W^{t, p}(\mathcal{O}),
$$

where $[\cdot]$ is a seminorm on $W^{t, p}(\mathcal{O})$, then

$$
|\zeta(w)| \leq C_{0} C_{1}(\operatorname{diam}(\mathcal{O}))^{t-s}[w]_{W^{t, p}(\mathcal{O})} \quad \forall w \in \mathbb{H} \cap W^{t, p}(\mathcal{O}) .
$$

We note that when $\mathcal{O}=K, \mathbb{S}=\mathbb{Q}_{1}(K)$ (with $\mathbb{Q}_{1}(K)$ as defined above when $d=2$ and a completely analogous definition when $d=3$; cf. Section 5), $s=0,1,1 \leq p \leq \infty$ and with $Q_{A} w \in \mathbb{Q}_{1}(K)$ signifying the averaged Taylor polynomial of a smooth function $w$ introduced in [11, Section 4.6], we have that

$$
\left\|w-Q_{A} w\right\|_{W^{s, p}(K)} \leq C_{1} \varepsilon^{2-s} \sum_{\alpha=1}^{d}\left\|\partial_{\alpha}^{2} w\right\|_{L^{p}(K)},
$$

where $C_{1}$ is a positive constant independent of $\varepsilon$. Due to the fact that we work on $\mathbb{Q}_{1}(K)$ the right-hand side of this estimate involves only part of the standard $W^{2, p}(K)$ seminorm, see [11, Section 4.6] for a proof and a related discussion. Thus, for a linear functional $\zeta$ that vanishes on $\mathbb{S}=\mathbb{Q}_{1}(K)$ and such that $|\zeta(w)| \leq C_{0}\|w\|_{L^{p}(K)}$ for all $w \in \mathbb{H}=C(\bar{K})$, we have from Lemma 2.1 with $s=0, t=2$ and $1 \leq p \leq \infty$ that

$$
|\zeta(w)| \leq C_{0} C_{1} \varepsilon^{2} \sum_{\alpha=1}^{d}\left\|\partial_{\alpha}^{2} w\right\|_{L^{p}(K)} \quad \forall w \in C(\bar{K}) \cap W^{2, p}(K) .
$$

Next, we observe that in $\mathbb{Q}_{1}(K)$ the following inverse inequality holds: for $1 \leq p \leq \infty$ and $1 \leq q \leq \infty$, there exists a constant $\gamma_{s, p, q}$ such that

$$
\|\varphi\|_{W^{s, q}(K)} \leq \gamma_{s, p, q} \varepsilon^{d / q-d / p}\|\varphi\|_{W^{s, p}(K)}, \quad \varphi \in \mathbb{Q}_{1}(K), \quad s=0,1 .
$$

For more general inverse inequalities involving variable degree derivatives we refer to [11, Section 4.5]. We note that (2.2) follows by a simple homogeneity argument on $\mathbb{Q}_{1}$. We will use the following consequence of (2.2): if $w \in \boldsymbol{V}_{\varepsilon}$, and $\alpha_{K} \geq 0$, then, for $1 / p+1 / q=1$,

$$
\begin{aligned}
& \sum_{K \in \mathcal{T}}|K| \alpha_{K}\left\|\partial_{\alpha} w\right\|_{L^{\infty}(K)} \leq \gamma_{1, p, \infty}\left\{\sum_{K \in \mathcal{T}}|K| \alpha_{K}^{q}\right\}^{1 / q}\|w\|_{W^{1, p}(\Omega)}, \quad w \in \boldsymbol{V}_{\varepsilon}, \quad q \neq \infty \\
& \sum_{K \in \mathcal{T}}|K| \alpha_{K}\left\|\partial_{\alpha} w\right\|_{L^{\infty}(K)} \leq \gamma_{1,1, \infty} \max _{K \in \mathcal{T}} \alpha_{K}\|w\|_{W^{1,1}(\Omega)}, \quad w \in \boldsymbol{V}_{\varepsilon}, \quad q=\infty .
\end{aligned}
$$

Indeed, these inequalities follow by Hölder' s inequality and by noting that, thanks to (2.2),

$$
\begin{aligned}
\left\{\sum_{K \in \mathcal{T}}|K|\left\|\partial_{\alpha} w\right\|_{L^{\infty}(K)}^{p}\right\}^{1 / p} & \leq\left\{\sum_{K \in \mathcal{T}}|K| \gamma_{1, p, \infty}^{p}\left(\varepsilon^{-d / p}\right)^{p}\|w\|_{W^{1, p}(K)}^{p}\right\}^{1 / p} \\
& =\gamma_{1, p, \infty}\|w\|_{W^{1, p}(\Omega)} .
\end{aligned}
$$


2.2. Consistency analysis of the Cauchy-Born model: $d=2$. Suppose that $v \in \boldsymbol{V}_{\varepsilon}$ and let $y$ be a sufficiently smooth function. Our aim is to approximate

$$
\left\langle D \Phi^{C B}(y), v\right\rangle=\int_{\Omega} S_{i \alpha}(\nabla y(x)) \frac{\partial v^{i}(x)}{\partial x_{\alpha}} \mathrm{d} x=\int_{\Omega} S_{i \alpha}(\nabla y(x)) \partial_{\alpha} v^{i}(x) \mathrm{d} x, \quad v \in \boldsymbol{V}_{\varepsilon} .
$$

It suffices to consider a (generic) term of the above sum. To simplify the notation we drop the vector indices and let $g(\nabla y)$ be the part that corresponds to the stress tensor. In the sequel we will consider

$$
\int_{\Omega} g(\nabla y(x)) \partial_{1} v(x) \mathrm{d} x, \quad v \in \boldsymbol{V}_{\varepsilon} .
$$

Recall that, since $v \in \boldsymbol{V}_{\varepsilon}$, we have that

$$
v(x)=\sum_{\ell \in \mathfrak{L}} v_{\left(\ell_{1}, \ell_{2}\right)} \Psi_{\ell_{1}}\left(x_{1}\right) \Psi_{\ell_{2}}\left(x_{2}\right), \quad \ell=\left(\ell_{1}, \ell_{2}\right) \in \mathfrak{L}, v_{\ell}=v\left(x_{\ell}\right) .
$$

Thus

$$
\begin{aligned}
\partial_{1} v(x) & =\sum_{\ell \in \mathfrak{L}} v_{\left(\ell_{1}, \ell_{2}\right)} \frac{1}{\varepsilon}\left(1_{\left[x_{\ell_{1}-1}, x_{\ell_{1}}\right]}\left(x_{1}\right)-1_{\left[x_{\ell_{1}}, x_{\ell_{1}+1}\right]}\left(x_{1}\right)\right) \Psi_{\ell_{2}}\left(x_{2}\right) \\
& =\sum_{\ell \in \mathfrak{L}} \frac{1}{\varepsilon}\left(v_{\left(\ell_{1}+1, \ell_{2}\right)}-v_{\left(\ell_{1}, \ell_{2}\right)}\right) 1_{\left[x_{\ell_{1}}, x_{\ell_{1}+1}\right]}\left(x_{1}\right) \Psi_{\ell_{2}}\left(x_{2}\right) .
\end{aligned}
$$

Therefore, by defining

$$
G(x):=G\left(x_{1}, x_{2}\right)=\int_{x_{-N_{1}-1}}^{x_{1}} g\left(\nabla y\left(x_{1}^{\prime}, x_{2}\right)\right) \mathrm{d} x_{1}^{\prime},
$$

we have that

$$
\begin{aligned}
\int_{\Omega} g( & \nabla y(x)) \partial_{1} v(x) \mathrm{d} x=\frac{1}{\varepsilon} \sum_{\ell \in \mathfrak{L}} \int_{\Omega} g(\nabla y(x))\left(v_{\left(\ell_{1}+1, \ell_{2}\right)}-v_{\left(\ell_{1}, \ell_{2}\right)}\right) 1_{\left[x_{\ell_{1}}, x_{\ell_{1}+1}\right]}\left(x_{1}\right) \Psi_{\ell_{2}}\left(x_{2}\right) \mathrm{d} x \\
= & \frac{1}{\varepsilon} \sum_{\ell \in \mathfrak{L}} \int_{x_{\ell_{2}-1}}^{x_{\ell_{2}+1}}\left\{\int_{x_{\ell_{1}}}^{x_{\ell_{1}+1}} g(\nabla y(x)) \mathrm{d} x_{1}\left[v_{\left(\ell_{1}+1, \ell_{2}\right)}-v_{\left(\ell_{1}, \ell_{2}\right)}\right] \Psi_{\ell_{2}}\left(x_{2}\right)\right\} \mathrm{d} x_{2} \\
= & \frac{1}{\varepsilon} \sum_{\ell \in \mathfrak{L}} \int_{x_{\ell_{2}-1}}^{x_{\ell_{2}+1}}\left[G\left(x_{\ell_{1}+1}, x_{2}\right)-G\left(x_{\ell_{1}}, x_{2}\right)\right] \Psi_{\ell_{2}}\left(x_{2}\right) \mathrm{d} x_{2}\left[v_{\left(\ell_{1}+1, \ell_{2}\right)}-v_{\left(\ell_{1}, \ell_{2}\right)}\right] \\
= & \frac{1}{\varepsilon} \sum_{\ell \in \mathfrak{L}} \int_{x_{\ell_{2}-1}}^{x_{\ell_{2}+1}}\left[G\left(x_{\ell_{1}+1}, x_{2}\right)-G\left(x_{\ell_{1}}, x_{2}\right)\right]\left(\Psi_{\ell_{2}}\left(x_{2}\right)-\frac{1}{2}\right) \mathrm{d} x_{2}\left[v_{\left(\ell_{1}+1, \ell_{2}\right)}-v_{\left(\ell_{1}, \ell_{2}\right)}\right] \\
& \quad+\frac{1}{\varepsilon} \sum_{\ell \in \mathfrak{L}} \int_{x_{\ell_{2}-1}}^{x_{\ell_{2}+1}}\left[G\left(x_{\ell_{1}+1}, x_{2}\right)-G\left(x_{\ell_{1}}, x_{2}\right)\right] \frac{1}{2} \mathrm{~d} x_{2}\left[v_{\left(\ell_{1}+1, \ell_{2}\right)}-v_{\left(\ell_{1}, \ell_{2}\right)}\right] \\
= & : A_{1}+A_{2} .
\end{aligned}
$$

In the next lemma we show that $A_{1}$ is second order accurate with respect to $\varepsilon$. We refer to [40] for a similar result.

Lemma 2.2. Let $y$ be a smooth function; then

$$
\left|\int_{x_{\ell_{2}-1}}^{x_{\ell_{2}+1}}\left[G\left(x_{\ell_{1}+1}, x_{2}\right)-G\left(x_{\ell_{1}}, x_{2}\right)\right]\left(\Psi_{\ell_{2}}\left(x_{2}\right)-\frac{1}{2}\right) \mathrm{d} x_{2}\right| \leq C(y)|K| \varepsilon^{2} .
$$


Proof. It suffices to consider the functional

$$
\zeta(G)=\int_{x_{\ell_{2}-1}}^{x_{\ell_{2}+1}}\left[G\left(x_{\ell_{1}+1}, x_{2}\right)-G\left(x_{\ell_{1}}, x_{2}\right)\right]\left(\Psi_{\ell_{2}}\left(x_{2}\right)-\frac{1}{2}\right) \mathrm{d} x_{2}, \quad G \in C\left(\overline{K \cup K_{e_{1}}}\right),
$$

where $K_{e_{1}}$ is the element that shares the edge $\left[x_{\ell}, x_{\ell+e_{1}}\right]$ with $K$, and to observe that

$$
\zeta(\varphi)=0 \quad \text { for all } \varphi \in \mathbb{P}_{2} .
$$

Indeed, if

$$
\begin{aligned}
& \varphi=x_{1}^{m} \quad \text { then } \quad \zeta(\varphi)=\left[\left(x_{\ell_{1}+1}\right)^{m}-\left(x_{\ell_{1}}\right)^{m}\right] \int_{x_{\ell_{2}-1}}^{x_{\ell_{2}+1}}\left(\Psi_{\ell_{2}}\left(x_{2}\right)-\frac{1}{2}\right) \mathrm{d} x_{2}=0, \quad \text { and if } \\
& \varphi=x_{2}^{m} \text { then } \quad \zeta(\varphi)=\int_{x_{\ell_{2}-1}}^{x_{\ell_{2}+1}}\left[\left(x_{2}\right)^{m}-\left(x_{2}\right)^{m}\right]\left(\Psi_{\ell_{2}}\left(x_{2}\right)-\frac{1}{2}\right) \mathrm{d} x_{2}=0 .
\end{aligned}
$$

Finally, for $\varphi=x_{1}\left(x_{2}-x_{\ell_{2}}\right)$,

$$
\zeta(\varphi)=\left[x_{\ell_{1}+1}-x_{\ell_{1}}\right] \int_{x_{\ell_{2}-1}}^{x_{\ell_{2}+1}}\left(x_{2}-x_{\ell_{2}}\right)\left(\Psi_{\ell_{2}}\left(x_{2}\right)-\frac{1}{2}\right) \mathrm{d} x_{2}=0 .
$$

Next we observe that by Lemma 2.1 we have, for any $\varphi \in \mathbb{P}_{2}$, that

$$
\begin{aligned}
|\zeta(G)| & \leq C \max _{x_{\ell_{1}} \leq s \leq x_{\ell_{1}+1}} \int_{x_{\ell_{2}-1}}^{x_{\ell_{2}+1}}\left|G\left(s, x_{2}\right)-\varphi\left(s, x_{2}\right)\right| \mathrm{d} x_{2} \\
& \leq C \varepsilon\|G-\varphi\|_{L^{\infty}\left(K \cup K_{e_{1}}\right)} \| \quad \forall G \in C\left(\overline{K \cup K_{e_{1}}}\right),
\end{aligned}
$$

The proof is completed by using standard approximation properties of $\mathbb{P}_{2}$ (see, [11]) to bound inf $\operatorname{liP}_{\varphi} \| G-$ $\varphi \|_{L^{\infty}\left(K \cup K_{e_{1}}\right)}$ by $C(y) \varepsilon^{3}$ and noting that $|K|=\left|K_{e_{1}}\right|=\varepsilon^{2}$.

The term $A_{2}$ requires further simplification. To this end, we note that

$$
\begin{aligned}
A_{2}= & \frac{1}{2 \varepsilon} \sum_{\ell \in \mathfrak{L}} \int_{x_{\ell_{2}-1}}^{x_{\ell_{2}+1}}\left[G\left(x_{\ell_{1}+1}, x_{2}\right)-G\left(x_{\ell_{1}}, x_{2}\right)\right] \mathrm{d} x_{2}\left[v_{\left(\ell_{1}+1, \ell_{2}\right)}-v_{\left(\ell_{1}, \ell_{2}\right)}\right] \\
= & \frac{1}{2 \varepsilon} \sum_{\ell \in \mathfrak{L}} \int_{x_{\ell_{2}-1}}^{x_{\ell_{2}+1}}\left\{\int_{x_{\ell_{1}}}^{x_{\ell_{1}+1}} g(\nabla y(x)) \mathrm{d} x_{1}\right\} \mathrm{d} x_{2}\left[v_{\left(\ell_{1}+1, \ell_{2}\right)}-v_{\left(\ell_{1}, \ell_{2}\right)}\right] \\
= & \frac{1}{2 \varepsilon} \sum_{\ell \in \mathfrak{L}} \int_{x_{\ell_{2}}}^{x_{\ell_{2}+1}}\left\{\int_{x_{\ell_{1}}}^{x_{\ell_{1}+1}} g(\nabla y(x)) \mathrm{d} x_{1}\right\} \mathrm{d} x_{2}\left[v_{\left(\ell_{1}+1, \ell_{2}\right)}-v_{\left(\ell_{1}, \ell_{2}\right)}\right] \\
& +\frac{1}{2 \varepsilon} \sum_{\ell^{\prime} \in \mathfrak{L}} \int_{x_{\ell_{2}^{\prime}}}^{x_{\ell_{2}^{\prime}+1}}\left\{\int_{x_{\ell_{1}}}^{x_{\ell_{1}+1}} g(\nabla y(x)) \mathrm{d} x_{1}\right\} \mathrm{d} x_{2}\left[v_{\left(\ell_{1}+1, \ell_{2}^{\prime}+1\right)}-v_{\left(\ell_{1}, \ell_{2}^{\prime}+1\right)}\right] \\
= & \sum_{K \in \mathcal{T}} \int_{K} g(\nabla y(x)) \mathrm{d} x \frac{1}{2}\left\{\bar{D}_{e_{1}} v_{\ell}+\bar{D}_{e_{1}} v_{\ell+e_{2}}\right\},
\end{aligned}
$$

where $\ell^{\prime}=\left(\ell_{1}, \ell_{2}^{\prime}\right)$. We shall also require the following result.

Lemma 2.3. Let $m_{K}$ be the barycenter of $K$; then, there exists a constant $C=C(y)$ such that

$$
\left|\sum_{K \in \mathcal{T}}\left[\int_{K} g(\nabla y(x)) \mathrm{d} x-|K| g\left(\nabla y\left(m_{K}\right)\right)\right] \frac{1}{2}\left\{\bar{D}_{e_{1}} v_{\ell}+\bar{D}_{e_{1}} v_{\ell+e_{2}}\right\}\right| \leq C(y) \varepsilon^{2}|v|_{W^{1, p}(\Omega)} .
$$


Proof. We denote by $\zeta$ the functional

$$
\zeta(w):=\frac{1}{|K|} \int_{K}\left\{w(x)-w\left(m_{K}\right)\right\} \mathrm{d} x
$$

and observe that

$$
\zeta(\varphi)=0 \quad \text { for all } \varphi \in \mathbb{Q}_{1}(K) .
$$

Obviously $|\zeta(w)| \leq 2\|w\|_{L^{\infty}(K)}$ for every $w \in C(\bar{K})$, and thus by Lemma 2.1 and (2.1) we have that

$$
|\zeta(w)| \leq C \varepsilon^{2} \sum_{\alpha=1}^{d}\left\|\partial_{\alpha}^{2} w\right\|_{L^{\infty}(K)} .
$$

Therefore, using the fact that $v \in \boldsymbol{V}_{\varepsilon}$ we deduce with $w(\cdot)=g(\nabla y(\cdot))$ that

$$
\begin{aligned}
& \mid \sum_{K \in \mathcal{T}}\left[\int_{K} g(\nabla y(x)) \mathrm{d} x-\mid\right.\left.K \mid g\left(\nabla y\left(m_{K}\right)\right)\right] \frac{1}{2}\left\{\bar{D}_{e_{1}} v_{\ell}+\bar{D}_{e_{1}} v_{\ell+e_{2}}\right\} \mid \\
& \leq C \varepsilon^{2} \sum_{K \in \mathcal{T}}|K|\left[\sum_{\alpha=1}^{d}\left\|\partial_{\alpha}^{2} g(\nabla y(x))\right\|_{L^{\infty}(K)}\right]\left\|\partial_{1} v\right\|_{L^{\infty}(K)} .
\end{aligned}
$$

The proof is thus complete in view of (2.3).

We summarize what we have shown so far in the following proposition.

Proposition 2.1. Suppose that $y$ is a smooth function, and let $m_{K}$ be the barycenter of $K$; then, for any $v \in \boldsymbol{V}_{\varepsilon}$, the quantity

$$
\begin{aligned}
\left\langle\mathcal{A}^{a, C B}, v\right\rangle_{\varepsilon}:= & \varepsilon^{d} \sum_{K \in \mathcal{T}} \sum_{\eta \in R}\left\{S_{i 1}\left(\nabla y\left(m_{K}\right)\right)\right\}\left[\frac{1}{2}\left\{\bar{D}_{e_{1}} v_{\ell}+\bar{D}_{e_{1}} v_{\ell+e_{2}}\right\}\right]_{i} \\
& +\varepsilon^{d} \sum_{K \in \mathcal{T}} \sum_{\eta \in R}\left\{S_{i 2}\left(\nabla y\left(m_{K}\right)\right)\right\}\left[\frac{1}{2}\left\{\bar{D}_{e_{2}} v_{\ell}+\bar{D}_{e_{2}} v_{\ell+e_{1}}\right\}\right]_{i} \\
= & \varepsilon^{d} \sum_{K \in \mathcal{T}} \sum_{\eta \in R}\left\{\nabla_{\zeta} \phi_{\eta}\left(\nabla y\left(m_{K}\right) \eta\right) \eta_{1}\right\} \frac{1}{2}\left\{\bar{D}_{e_{1}} v_{\ell}+\bar{D}_{e_{1}} v_{\ell+e_{2}}\right\} \\
& +\varepsilon^{d} \sum_{K \in \mathcal{T}} \sum_{\eta \in R}\left\{\nabla_{\zeta} \phi_{\eta}\left(\nabla y\left(m_{K}\right) \eta\right) \eta_{2}\right\} \frac{1}{2}\left\{\bar{D}_{e_{2}} v_{\ell}+\bar{D}_{e_{2}} v_{\ell+e_{1}}\right\},
\end{aligned}
$$

is a second order approximation to $\left\langle D \Phi^{C B}(y), v\right\rangle$ in the sense that there exists a constant $M=M(y, p)$, $1 \leq p \leq \infty$, independent of $v$, such that

$$
\left|\left\langle D \Phi^{C B}(y), v\right\rangle-\left\langle\mathcal{A}^{a, C B}, v\right\rangle_{\varepsilon}\right| \leq M \varepsilon^{2}|v|_{W^{1, p}(\Omega)} .
$$

2.3. The atomistic Cauchy-Born model. We define the average difference quotient (discrete derivative) as follows:

$$
\begin{aligned}
& \overline{\bar{D}}_{e_{1}} v_{\ell}:=\frac{1}{2}\left\{\bar{D}_{e_{1}} v_{\ell}+\bar{D}_{e_{1}} v_{\ell+e_{2}}\right\}, \\
& \overline{\bar{D}}_{e_{2}} v_{\ell}:=\frac{1}{2}\left\{\bar{D}_{e_{2}} v_{\ell}+\bar{D}_{e_{2}} v_{\ell+e_{1}}\right\} .
\end{aligned}
$$

Thus we can define the discrete gradient matrix as

$$
\left\{\bar{\nabla} v_{\ell}\right\}_{i \alpha}=\overline{\bar{D}}_{e_{\alpha}} v_{\ell}^{i}
$$


We introduce the atomistic potential

$$
\begin{aligned}
\Phi^{a, C B}(y) & :=\varepsilon^{d} \sum_{\ell \in \mathfrak{L}} \sum_{\eta \in R} \phi_{\eta}\left(\bar{\nabla} y_{\ell} \eta\right) \\
& =\varepsilon^{d} \sum_{\ell \in \mathfrak{L}} W_{C B}\left(\bar{\nabla} y_{\ell}\right) .
\end{aligned}
$$

Notice that due to the definitions of $\overline{\bar{D}}_{e_{\alpha}} v_{\ell}, \alpha=1,2$, as averages of discrete gradients, $\Phi^{a, C B}$ is not the standard atomistic potential that one gets by replacing in the atomistic model long-range interactions by short-range ones. In fact, in that case the corresponding atomistic potential would be

$$
\begin{aligned}
\widetilde{\Phi}^{a, C B}(y) & :=\varepsilon^{d} \sum_{\ell \in \mathfrak{L}} \sum_{\eta \in R} \phi_{\eta}\left(\widetilde{\nabla} y_{\ell} \eta\right) \\
& =\varepsilon^{d} \sum_{\ell \in \mathfrak{L}} W_{C B}\left(\widetilde{\nabla} y_{\ell}\right), \quad \text { where }\left\{\widetilde{\nabla} y_{\ell}\right\}_{i \alpha}=\bar{D}_{e_{\alpha}} y_{\ell}^{i}
\end{aligned}
$$

Now, for a given field of external forces $f: \mathfrak{L} \rightarrow \mathbb{R}^{d}$ the atomistic Cauchy-Born problem reads:

$$
\text { find a local minimizer } y^{a, C B} \text { in } \mathcal{X} \text { of : }
$$

$$
\Phi^{a, C B}(y)-\langle f, y\rangle_{\varepsilon} \text {. }
$$

If such a minimizer exists, then

$$
\left\langle D \Phi^{a, C B}\left(y^{a, C B}\right), v\right\rangle_{\varepsilon}=\langle f, v\rangle_{\varepsilon}, \quad \text { for all } v \in \mathscr{V} .
$$

The next lemma provides the link between $\mathcal{A}^{a, C B}$ introduced earlier in Proposition 2.1 and $D \Phi^{a, C B}$.

Lemma 2.4. Let $y \in \boldsymbol{V}_{\varepsilon}$; then for, any $v \in \boldsymbol{V}_{\varepsilon}$,

$$
\left\langle\mathcal{A}^{a, C B}, v\right\rangle_{\varepsilon}=\left\langle D \Phi^{a, C B}(y), v\right\rangle_{\varepsilon}
$$

Proof. Obviously,

$$
\begin{aligned}
\left\langle D \Phi^{a, C B}(y), v\right\rangle_{\varepsilon} & =\varepsilon^{d} \sum_{\ell \in \mathfrak{L}} \sum_{\eta \in R}\left\{S_{i 1}\left(\bar{\nabla} y_{\ell}\right)\right\}\left[\frac{1}{2}\left\{\bar{D}_{e_{1}} v_{\ell}+\bar{D}_{e_{1}} v_{\ell+e_{2}}\right\}\right]_{i} \\
& +\varepsilon^{d} \sum_{\ell \in \mathfrak{L}} \sum_{\eta \in R}\left\{S_{i 2}\left(\bar{\nabla} y_{\ell}\right)\right\}\left[\frac{1}{2}\left\{\bar{D}_{e_{2}} v_{\ell}+\bar{D}_{e_{2}} v_{\ell+e_{1}}\right\}\right]_{i} .
\end{aligned}
$$

Hence, it suffices to observe that for $y \in \boldsymbol{V}_{\varepsilon}$ and for $K$ being the element with vertices $x_{\ell}, x_{\ell+e_{1}}, x_{\ell+e_{1}+e_{2}}$, $x_{\ell+e_{2}}$, see Fig. 1, we have that

$$
\nabla y\left(m_{K}\right)=\bar{\nabla} y_{\ell}, \quad \text { for } y \in \boldsymbol{V}_{\varepsilon},
$$

and thus $S_{i \alpha}\left(\nabla y\left(m_{K}\right)\right)=S_{i \alpha}\left(\bar{\nabla} y_{\ell}\right)$. 


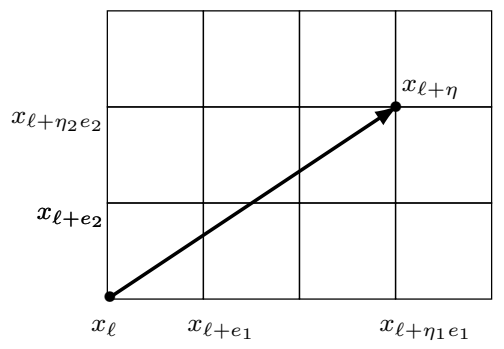

FIGURE 1. Typical interaction vector $\eta$ and the corresponding lattice points.

\section{Comparison of Atomistic CAUChy-Born And ATOMistic models: $d=2$}

To compare the atomistic and atomistic Cauchy-Born models we start from (1.4) and note that:

$$
\begin{aligned}
\left\langle D \Phi^{a}(y), v\right\rangle_{\varepsilon}= & \varepsilon^{d} \sum_{\ell \in \mathfrak{L}} \sum_{\eta \in R} \nabla_{\zeta} \phi_{\eta}\left(\bar{D}_{\eta} y_{\ell}\right) \cdot \bar{D}_{\eta} v_{\ell} \\
= & \varepsilon^{d} \sum_{\ell \in \mathfrak{L}} \sum_{\eta \in R} \nabla_{\zeta} \phi_{\eta}\left(\bar{D}_{\eta} y_{\ell}\right) \cdot\left\{\frac{1}{2} \bar{D}_{\eta_{1} e_{1}} v_{\ell}+\frac{1}{2} \bar{D}_{\eta_{1} e_{1}} v_{\ell+\eta_{2} e_{2}}\right\} \\
& +\varepsilon^{d} \sum_{\ell \in \mathfrak{L}} \sum_{\eta \in R} \nabla_{\zeta} \phi_{\eta}\left(\bar{D}_{\eta} y_{\ell}\right) \cdot\left\{\frac{1}{2} \bar{D}_{\eta_{2} e_{2}} v_{\ell}+\frac{1}{2} \bar{D}_{\eta_{2} e_{2}} v_{\ell+\eta_{1} e_{1}}\right\} .
\end{aligned}
$$

Due to the assumed periodicity of the lattice, we have that

$$
\begin{aligned}
\left\langle D \Phi^{a}(y), v\right\rangle_{\varepsilon}= & \varepsilon^{d} \sum_{\ell \in \mathfrak{L}} \sum_{\eta \in R} \nabla_{\zeta} \phi_{\eta}\left(\bar{D}_{\eta} y_{\ell}\right) \cdot \bar{D}_{\eta} v_{\ell} \\
= & \varepsilon^{d} \sum_{\ell \in \mathfrak{L}} \sum_{\eta \in R}\left\{\frac{1}{2} \nabla_{\zeta} \phi_{\eta}\left(\bar{D}_{\eta} y_{\ell}\right)+\frac{1}{2} \nabla_{\zeta} \phi_{\eta}\left(\bar{D}_{\eta} y_{\ell-\eta_{2} e_{2}}\right)\right\} \cdot \bar{D}_{\eta_{1} e_{1}} v_{\ell} \\
& +\varepsilon^{d} \sum_{\ell \in \mathfrak{L}} \sum_{\eta \in R}\left\{\frac{1}{2} \nabla_{\zeta} \phi_{\eta}\left(\bar{D}_{\eta} y_{\ell}\right)+\frac{1}{2} \nabla_{\zeta} \phi_{\eta}\left(\bar{D}_{\eta} y_{\ell-\eta_{1} e_{1}}\right)\right\} \cdot \bar{D}_{\eta_{2} e_{2}} v_{\ell} .
\end{aligned}
$$

Notice that when $\eta_{1}, \eta_{2}$ are positive,

$$
\begin{aligned}
& \bar{D}_{\eta_{1} e_{1}} v_{\ell}=\bar{D}_{e_{1}} v_{\ell}+\cdots+\bar{D}_{e_{1}} v_{\ell+\left(\eta_{1}-1\right) e_{1}}, \\
& \bar{D}_{\eta_{2} e_{2}} v_{\ell}=\bar{D}_{e_{2}} v_{\ell}+\cdots+\bar{D}_{e_{2}} v_{\ell+\left(\eta_{2}-1\right) e_{2}}
\end{aligned}
$$

while when, e.g., $\eta_{1}=-\sigma_{1}<0$,

$$
\bar{D}_{\eta_{1} e_{1}} v_{\ell}=-\bar{D}_{e_{1}} v_{\ell-\sigma_{1} e_{1}}-\cdots-\bar{D}_{e_{1}} v_{\ell-e_{1}} .
$$

Therefore, we deduce that

$$
\left\langle D \Phi^{a}(y), v\right\rangle_{\varepsilon}=\varepsilon^{d} \sum_{\ell \in \mathfrak{L}} \sum_{\eta \in R} \bar{\Phi}_{\eta, \ell, 1} \cdot \bar{D}_{e_{1}} v_{\ell}+\varepsilon^{d} \sum_{\ell \in \mathfrak{L}} \sum_{\eta \in R} \bar{\Phi}_{\eta, \ell, 2} \cdot \bar{D}_{e_{2}} v_{\ell}
$$


where

$$
\bar{\Phi}_{\eta, \ell, 2}:= \begin{cases}\sum_{k=0}^{\eta_{2}-1}\left\{\frac{1}{2} \nabla_{\zeta} \phi_{\eta}\left(\bar{D}_{\eta} y_{\ell-k e_{2}}\right)+\frac{1}{2} \nabla_{\zeta} \phi_{\eta}\left(\bar{D}_{\eta} y_{\ell-k e_{2}-\eta_{1} e_{1}}\right)\right\}, & \text { for } \eta_{2}>0 \\ \sum_{k=1}^{\sigma_{2}}-\left\{\frac{1}{2} \nabla_{\zeta} \phi_{\eta}\left(\bar{D}_{\eta} y_{\ell+k e_{2}}\right)+\frac{1}{2} \nabla_{\zeta} \phi_{\eta}\left(\bar{D}_{\eta} y_{\ell+k e_{2}-\eta_{1} e_{1}}\right)\right\}, & \text { for } \eta_{2}=-\sigma_{2}<0,\end{cases}
$$

and $\bar{\Phi}_{\eta, \ell, 1}$ is defined analogously.

In the sequel, we focus on the term $\bar{\Phi}_{\eta, \ell, 2}$ when $\eta_{2}>0$. The other terms are treated in a similar manner. As a first step we will compare $\bar{\Phi}_{\eta, \ell, 2}$ with

$$
\Phi_{\eta, \ell, 2}:=\sum_{k=0}^{\eta_{2}-1} \nabla_{\zeta} \phi_{\eta}\left(\nabla y\left(m_{k, \eta_{2}, \ell}\right) \eta\right), \quad \eta_{2}>0
$$

where

$$
\begin{array}{ll}
m_{k, \eta_{2}, \ell} & \text { is the midpoint of the side with endpoints } x_{\ell-k e_{2}}, x_{\ell-k e_{2}+\eta_{2} e_{2}}, \\
m_{2, \ell} & \text { is the midpoint of the side with endpoints } x_{\ell}, x_{\ell+e_{2}}, \text { and } \\
m_{1, \ell} & \text { is the midpoint of the side with endpoints } x_{\ell}, x_{\ell+e_{1}} .
\end{array}
$$

We have the following result.

Lemma 3.1. Let $y$ be a smooth function; then, assuming that $\eta_{2}>0$, we have that

$$
\left|\bar{\Phi}_{\eta, \ell, 2}-\Phi_{\eta, \ell, 2}\right| \leq C(y) \varepsilon^{2} .
$$

Proof. We begin by noting that (in the case of $k=0$ ):

$$
\begin{aligned}
& \frac{1}{2} \nabla_{\zeta} \phi_{\eta}\left(\bar{D}_{\eta} y_{\ell}\right)+\frac{1}{2} \nabla_{\zeta} \phi_{\eta}\left(\bar{D}_{\eta} y_{\ell-\eta_{1} e_{1}}\right) \\
& =\frac{1}{2} \nabla_{\zeta} \phi_{\eta}\left(\left\{\frac{1}{2} \bar{D}_{\eta_{1} e_{1}} y_{\ell}+\frac{1}{2} \bar{D}_{\eta_{1} e_{1}} y_{\ell+\eta_{2} e_{2}}\right\}+\left\{\frac{1}{2} \bar{D}_{\eta_{2} e_{2}} y_{\ell}+\frac{1}{2} \bar{D}_{\eta_{2} e_{2}} y_{\ell+\eta_{1} e_{1}}\right\}\right) \\
& \quad+\frac{1}{2} \nabla_{\zeta} \phi_{\eta}\left(\left\{\frac{1}{2} \bar{D}_{\eta_{1} e_{1}} y_{\ell-\eta_{1} e_{1}}+\frac{1}{2} \bar{D}_{\eta_{1} e_{1}} y_{\ell-\eta_{1} e_{1}+\eta_{2} e_{2}}\right\}+\left\{\frac{1}{2} \bar{D}_{\eta_{2} e_{2}} y_{\ell-\eta_{1} e_{1}}+\frac{1}{2} \bar{D}_{\eta_{2} e_{2}} y_{\ell-\eta_{1} e_{1}+\eta_{1} e_{1}}\right\}\right) .
\end{aligned}
$$

We shall use the following elementary bound: there exists a constant $c_{1}=c_{1}\left(\psi^{\prime \prime}\right)$ such that, for any smooth function $\psi$ and real numbers $a, b$,

$$
\left|\frac{1}{2} \psi(a)+\frac{1}{2} \psi(b)-\psi\left(\frac{a+b}{2}\right)\right| \leq c_{1}|a-b|^{2} .
$$

We notice that

$$
\begin{aligned}
& \mid\left\{\frac{1}{2} \bar{D}_{\eta_{1} e_{1}} y_{\ell}+\frac{1}{2} \bar{D}_{\eta_{1} e_{1}} y_{\ell+\eta_{2} e_{2}}\right\}+\left\{\frac{1}{2} \bar{D}_{\eta_{2} e_{2}} y_{\ell}+\frac{1}{2} \bar{D}_{\eta_{2} e_{2}} y_{\ell+\eta_{1} e_{1}}\right\} \\
& \quad-\left\{\frac{1}{2} \bar{D}_{\eta_{1} e_{1}} y_{\ell-\eta_{1} e_{1}}+\frac{1}{2} \bar{D}_{\eta_{1} e_{1}} y_{\ell-\eta_{1} e_{1}+\eta_{2} e_{2}}\right\}-\left\{\frac{1}{2} \bar{D}_{\eta_{2} e_{2}} y_{\ell-\eta_{1} e_{1}}+\frac{1}{2} \bar{D}_{\eta_{2} e_{2}} y_{\ell-\eta_{1} e_{1}+\eta_{1} e_{1}}\right\} \mid \leq C \varepsilon .
\end{aligned}
$$

In view of (3.5) it suffices to consider

$$
\begin{aligned}
& \nabla_{\zeta} \phi_{\eta}\left(\left\{\frac{1}{4} \bar{D}_{\eta_{1} e_{1}} y_{\ell}+\frac{1}{4} \bar{D}_{\eta_{1} e_{1}} y_{\ell+\eta_{2} e_{2}}\right\}+\left\{\frac{1}{4} \bar{D}_{\eta_{2} e_{2}} y_{\ell}+\frac{1}{4} \bar{D}_{\eta_{2} e_{2}} y_{\ell+\eta_{1} e_{1}}\right\}\right. \\
& \left.\quad+\left\{\frac{1}{4} \bar{D}_{\eta_{1} e_{1}} y_{\ell-\eta_{1} e_{1}}+\frac{1}{4} \bar{D}_{\eta_{1} e_{1}} y_{\ell-\eta_{1} e_{1}+\eta_{2} e_{2}}\right\}+\left\{\frac{1}{4} \bar{D}_{\eta_{2} e_{2}} y_{\ell-\eta_{1} e_{1}}+\frac{1}{4} \bar{D}_{\eta_{2} e_{2}} y_{\ell-\eta_{1} e_{1}+\eta_{1} e_{1}}\right\}\right) \\
& =\nabla_{\zeta} \phi_{\eta}\left(\left\{\frac{1}{4} \bar{D}_{2 \eta_{1} e_{1}} y_{\ell-\eta_{1} e_{1}}+\frac{1}{4} \bar{D}_{2 \eta_{1} e_{1}} y_{\ell-\eta_{1} e_{1}+\eta_{2} e_{2}}\right\}\right. \\
& \left.\quad+\left\{\frac{1}{2} \bar{D}_{\eta_{2} e_{2}} y_{\ell}+\frac{1}{4} \bar{D}_{\eta_{2} e_{2}} y_{\ell+\eta_{1} e_{1}}+\frac{1}{4} \bar{D}_{\eta_{2} e_{2}} y_{\ell-\eta_{1} e_{1}}\right\}\right) .
\end{aligned}
$$




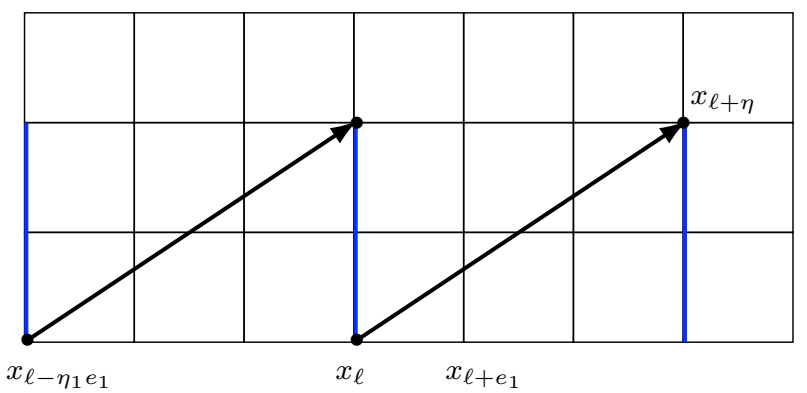

FIGURE 2. Symmetry as a result of combining $\bar{D}_{\eta} y_{\ell}$ and $\bar{D}_{\eta} y_{\ell-\eta_{1} e_{1}}$.

Now,

$$
\begin{aligned}
\mid\left\{\frac{1}{4} \bar{D}_{2 \eta_{1} e_{1}} y_{\ell-\eta_{1} e_{1}}+\right. & \left.\frac{1}{4} \bar{D}_{2 \eta_{1} e_{1}} y_{\ell-\eta_{1} e_{1}+\eta_{2} e_{2}}\right\}-\eta_{1} \partial_{1} y\left(m_{0, \eta_{2}, \ell}\right) \mid \\
\leq & \left|\frac{1}{2} \eta_{1} \partial_{1} y\left(x_{\ell}\right)+\frac{1}{2} \eta_{1} \partial_{1} y\left(x_{\ell+\eta_{2} e_{2}}\right)-\eta_{1} \partial_{1} y\left(m_{0, \eta_{2}, \ell}\right)\right| \\
& +\left|\frac{1}{4} \bar{D}_{2 \eta_{1} e_{1}} y_{\ell-\eta_{1} e_{1}}-\frac{1}{2} \eta_{1} \partial_{1} y\left(x_{\ell}\right)\right|+\left|\frac{1}{4} \bar{D}_{2 \eta_{1} e_{1}} y_{\ell-\eta_{1} e_{1}+\eta_{2} e_{2}}-\frac{1}{2} \eta_{1} \partial_{1} y\left(x_{\ell+\eta_{2} e_{2}}\right)\right| \\
\leq & C \varepsilon^{2} .
\end{aligned}
$$

Similarly,

$$
\left|\left\{\frac{1}{2} \bar{D}_{\eta_{2} e_{2}} y_{\ell}+\frac{1}{4} \bar{D}_{\eta_{2} e_{2}} y_{\ell+\eta_{1} e_{1}}+\frac{1}{4} \bar{D}_{\eta_{2} e_{2}} y_{\ell-\eta_{1} e_{1}}\right\}-\eta_{2} \partial_{2} y\left(m_{0, \eta_{2}, \ell}\right)\right| \leq C \varepsilon^{2} .
$$

Proceeding as above for any $k, k \leq \eta_{2}-1$, we complete the proof.

Next we establish the following result, which is valid for all positive or negative $\eta_{2}$.

Lemma 3.2. Let $y$ be a smooth function, and let $m_{2, \ell}$ denote the midpoint of the side with endpoints $x_{\ell}, x_{\ell+e_{2}}$ (cf. (3.4) above); then,

$$
\left|\eta_{2} \nabla_{\zeta} \phi_{\eta}\left(\nabla y\left(m_{2, \ell}\right) \eta\right)-\bar{\Phi}_{\eta, \ell, 2}\right| \leq C(y) \varepsilon^{2} .
$$

Proof. Assume first that $\eta_{2}>0$. It is a simple matter to observe that $\Phi_{\eta, \ell, 2}$ is a sum of $\eta_{2}$ terms involving function evaluations at points that are symmetrically placed with respect to $m_{2, \ell}$ (if $\eta_{2}$ is odd, one of these points is $m_{2, \ell}$ ). Thus,

$$
\left|\frac{1}{\eta_{2}} \Phi_{\eta, \ell, 2}-\nabla_{\zeta} \phi_{\eta}\left(\nabla y\left(m_{2, \ell}\right) \eta\right)\right| \leq C(y) \varepsilon^{2} .
$$

In the case where $\eta_{2}=-\sigma_{2}<0$ one can show that

$$
\left|-\sum_{k=1}^{\sigma_{2}} \nabla_{\zeta} \phi_{\eta}\left(\nabla y\left(m_{-k, \eta_{2}, \ell}\right) \eta\right)-\bar{\Phi}_{\eta, \ell, 2}\right| \leq C(y) \varepsilon^{2} .
$$


As before, the points $m_{-k, \eta_{2}, \ell}$ are symmetrically placed with respect to $m_{2, \ell}$. Hence

$$
\left|\frac{1}{\sigma_{2}} \sum_{k=1}^{\sigma_{2}} \nabla_{\zeta} \phi_{\eta}\left(\nabla y\left(m_{-k, \eta_{2}, \ell}\right) \eta\right)-\nabla_{\zeta} \phi_{\eta}\left(\nabla y\left(m_{2, \ell}\right) \eta\right)\right| \leq C(y) \varepsilon^{2}
$$

and the proof is complete.

We have therefore completed the proof of the following result.

Proposition 3.1. Let $y$ be a smooth function, and let $m_{1, \ell}, m_{2, \ell}$ be as in (3.4); then, for any $v \in \boldsymbol{V}_{\varepsilon}$, the quantity

$$
\begin{aligned}
\langle\mathcal{A}, v\rangle_{\varepsilon}:= & \varepsilon^{d} \sum_{\ell \in \mathfrak{L}} \sum_{\eta \in R}\left\{\nabla_{\zeta} \phi_{\eta}\left(\nabla y\left(m_{1, \ell}\right) \eta\right) \eta_{1}\right\} \cdot \bar{D}_{e_{1}} v_{\ell} \\
& +\varepsilon^{d} \sum_{\ell \in \mathfrak{L}} \sum_{\eta \in R}\left\{\nabla_{\zeta} \phi_{\eta}\left(\nabla y\left(m_{2, \ell}\right) \eta\right) \eta_{2}\right\} \cdot \bar{D}_{e_{2}} v_{\ell}
\end{aligned}
$$

is a second order approximation to $\left\langle D \Phi^{a}(y), v\right\rangle_{\varepsilon}$ in the sense that there exists a constant $M=M(y, p)$, independent of $v$, such that

$$
\left|\left\langle D \Phi^{a}(y), v\right\rangle_{\varepsilon}-\langle\mathcal{A}, v\rangle_{\varepsilon}\right| \leq M \varepsilon^{2}|v|_{W^{1, p}(\Omega)}, \quad 1 \leq p \leq \infty
$$

\section{ENERGY AND VARIATIONAL CONSISTENCY: $d=2$}

In this section we present our basic results in two space dimensions. Similar results for the comparison of atomistic and atomistic Cauchy-Born models, or atomistic Cauchy-Born and continuum Cauchy-Born models are proved in a similar fashion, however we omit their (entirely similar) statements.

Theorem 4.1. (VARIATIONAL CONSISTENCY) Let $y$ be a smooth function; then, for any $v \in \boldsymbol{V}_{\varepsilon}$, the continuum Cauchy-Born variation $\left\langle D \Phi^{C B}(y), v\right\rangle$ is a second order approximation to the atomistic variation $\left\langle D \Phi^{a}(y), v\right\rangle_{\varepsilon}$ in the sense that there exists a constant $M_{V}=M_{V}(y, p), 1 \leq p \leq \infty$, independent of $v$, such that

$$
\left|\left\langle D \Phi^{C B}(y)-\left\langle D \Phi^{a}(y), v\right\rangle_{\varepsilon}, v\right\rangle\right| \leq M_{V} \varepsilon^{2}|v|_{W^{1, p}(\Omega)} .
$$

Proof. If $x_{\ell}$ is the bottom-left vertex of $K$, we denote by $K_{e_{1}}$ the element that shares the edge $\left[x_{\ell}, x_{\ell+e_{1}}\right]$ with $K$. Similarly, we denote by $K_{e_{2}}$ the element that shares the edge $\left[x_{\ell}, x_{\ell+e_{2}}\right]$ with $K$. Then, one can rewrite (2.7) as

$$
\begin{aligned}
\left\langle\mathcal{A}^{a, C B}, v\right\rangle_{\varepsilon}:= & \varepsilon^{d} \sum_{K \in \mathcal{T}} \sum_{\eta \in R} \frac{1}{2}\left\{\nabla_{\zeta} \phi_{\eta}\left(\nabla y\left(m_{K}\right) \eta\right) \eta_{1}+\nabla_{\zeta} \phi_{\eta}\left(\nabla y\left(m_{K_{e_{1}}}\right) \eta\right) \eta_{1}\right\} \cdot \bar{D}_{e_{1}} v_{\ell} \\
& +\varepsilon^{d} \sum_{K \in \mathcal{T}} \sum_{\eta \in R} \frac{1}{2}\left\{\nabla_{\zeta} \phi_{\eta}\left(\nabla y\left(m_{K}\right) \eta\right) \eta_{2}+\nabla_{\zeta} \phi_{\eta}\left(\nabla y\left(m_{K_{e_{2}}}\right) \eta\right) \eta_{2}\right\} \cdot \bar{D}_{e_{2}} v_{\ell} .
\end{aligned}
$$

One should compare directly the above expression with (3.6). Since, obviously,

$$
\left|\frac{1}{2}\left\{\nabla_{\zeta} \phi_{\eta}\left(\nabla y\left(m_{K}\right) \eta\right) \eta_{1}+\nabla_{\zeta} \phi_{\eta}\left(\nabla y\left(m_{K_{e_{1}}}\right) \eta\right) \eta_{1}\right\}-\left\{\nabla_{\zeta} \phi_{\eta}\left(\nabla y\left(m_{1, \ell}\right) \eta\right) \eta_{1}\right\}\right| \leq C(y) \varepsilon^{2}
$$

the proof is complete in view of Propositions 2.1 and 3.1.

Theorem 4.2. (ENERGy CONSISTENCY) Let y be a smooth function; then, the continuum Cauchy-Born energy $\Phi^{C B}(y)$ is a second order approximation to the atomistic energy $\Phi^{a}(y)$ in the sense that there exists a constant $M_{E}=M_{E}(y)$, such that

$$
\left|\Phi^{C B}(y)-\Phi^{a}(y)\right| \leq M_{E} \varepsilon^{2} .
$$


Proof. The proof is similar to, though considerably simpler than, the proof of the variational consistency. We start from the continuum Cauchy-Born energy

$$
\begin{aligned}
\Phi^{C B}(y) & =\int_{\Omega} W_{C B}(\nabla y(x)) \mathrm{d} x=\sum_{K \in \mathcal{T}} \int_{K} W_{C B}(\nabla y(x)) \mathrm{d} x \\
& =\sum_{K \in \mathcal{T}}|K| W_{C B}\left(\nabla y\left(m_{K}\right)\right)+\sum_{K \in \mathcal{T}} \int_{K}\left[W_{C B}(\nabla y(x))-W_{C B}\left(\nabla y\left(m_{K}\right)\right)\right] \mathrm{d} x=: I_{1}+I_{2},
\end{aligned}
$$

where as before $m_{K}$ is the barycenter of $K$. Using similar arguments as in the proof of Lemma 2.3 we get

$$
\left|I_{2}\right| \leq C(y) \varepsilon^{2} \text {. }
$$

As before we will compare $I_{1}$ with the atomistic energy $\Phi^{a}(y)$. Since $m_{K}$ is the barycenter of $K$ the key point here is to rearrange the terms in $\Phi^{a}(y)$ in order to create symmetries around the cell $K$. In fact, using the assumed periodicity of the lattice, we have that

$$
\begin{aligned}
\Phi^{a}(y)= & \varepsilon^{d} \sum_{\ell \in \mathfrak{L}} \sum_{\eta \in R} \phi_{\eta}\left(\bar{D}_{\eta} y_{\ell}\right) \\
= & \varepsilon^{d} \sum_{\ell \in \mathfrak{L}} \sum_{\eta \in R} \frac{1}{4}\left[\phi_{\eta}\left(\bar{D}_{\eta} y_{\ell}\right)+\phi_{\eta}\left(\bar{D}_{\eta} y_{\ell-\left(\eta_{1}-1\right) e_{1}}\right)\right. \\
& +\phi_{\eta}\left(\bar{D}_{\eta} y_{\ell-\left(\eta_{2}-1\right) e_{2}}\right)+\phi_{\eta}\left(\bar{D}_{\eta} y_{\left.\left.\ell-\left(\eta_{1}-1\right) e_{1}-\left(\eta_{2}-1\right) e_{2}\right)\right] .}\right.
\end{aligned}
$$

Next we use similar splittings as in Lemma 3.1:

$$
\begin{aligned}
& \bar{D}_{\eta} y_{\ell}=\left\{\frac{1}{2} \bar{D}_{\eta_{1} e_{1}} y_{\ell}+\frac{1}{2} \bar{D}_{\eta_{1} e_{1}} y_{\ell+\eta_{2} e_{2}}\right\}+\left\{\frac{1}{2} \bar{D}_{\eta_{2} e_{2}} y_{\ell}+\frac{1}{2} \bar{D}_{\eta_{2} e_{2}} y_{\ell+\eta_{1} e_{1}}\right\} \text {, } \\
& \bar{D}_{\eta} y_{\ell-\left(\eta_{1}-1\right) e_{1}}=\left\{\frac{1}{2} \bar{D}_{\eta_{1} e_{1}} y_{\ell-\left(\eta_{1}-1\right) e_{1}}+\frac{1}{2} \bar{D}_{\eta_{1} e_{1}} y_{\ell-\left(\eta_{1}-1\right) e_{1}+\eta_{2} e_{2}}\right\} \\
& +\left\{\frac{1}{2} \bar{D}_{\eta_{2} e_{2}} y_{\ell-\left(\eta_{1}-1\right) e_{1}}+\frac{1}{2} \bar{D}_{\eta_{2} e_{2}} y_{\ell-\left(\eta_{1}-1\right) e_{1}+\eta_{1} e_{1}}\right\}, \\
& \bar{D}_{\eta} y_{\ell-\left(\eta_{2}-1\right) e_{2}}=\left\{\frac{1}{2} \bar{D}_{\eta_{1} e_{1}} y_{\ell-\left(\eta_{2}-1\right) e_{2}}+\frac{1}{2} \bar{D}_{\eta_{1} e_{1}} y_{\ell-\left(\eta_{2}-1\right) e_{2}+\eta_{2} e_{2}}\right\} \\
& +\left\{\frac{1}{2} \bar{D}_{\eta_{2} e_{2}} y_{\ell-\left(\eta_{2}-1\right) e_{2}}+\frac{1}{2} \bar{D}_{\eta_{2} e_{2}} y_{\ell-\left(\eta_{2}-1\right) e_{2}+\eta_{1} e_{1}}\right\}, \\
& \bar{D}_{\eta} y_{\ell-\left(\eta_{1}-1\right) e_{1}-\left(\eta_{2}-1\right) e_{2}}=\left\{\frac{1}{2} \bar{D}_{\eta_{1} e_{1}} y_{\ell-\left(\eta_{1}-1\right) e_{1}-\left(\eta_{2}-1\right) e_{2}}+\frac{1}{2} \bar{D}_{\eta_{1} e_{1}} y_{\ell-\left(\eta_{1}-1\right) e_{1}-\left(\eta_{2}-1\right) e_{2}+\eta_{2} e_{2}}\right\} \\
& +\left\{\frac{1}{2} \bar{D}_{\eta_{2} e_{2}} y_{\ell-\left(\eta_{1}-1\right) e_{1}-\left(\eta_{2}-1\right) e_{2}}+\frac{1}{2} \bar{D}_{\eta_{2} e_{2}} y_{\ell-\left(\eta_{1}-1\right) e_{1}-\left(\eta_{2}-1\right) e_{2}+\eta_{1} e_{1}}\right\} \text {. }
\end{aligned}
$$

Observe now that, for example,

$$
\begin{aligned}
\frac{1}{2} \bar{D}_{\eta_{1} e_{1}} y_{\ell} & +\frac{1}{2} \bar{D}_{\eta_{1} e_{1}} y_{\ell-\left(\eta_{1}-1\right) e_{1}}=\frac{y_{\ell+\eta_{1} e_{1}}-y_{\ell-\left(\eta_{1}-1\right) e_{1}}}{2 \varepsilon}+\frac{y_{\ell+e_{1}}-y_{\ell}}{2 \varepsilon} \\
= & \frac{2 \eta_{1}-1}{2} \partial_{1} y\left(m_{1, \ell}\right)+\frac{1}{2} \partial_{1} y\left(m_{1, \ell}\right)+\mathcal{O}\left(\varepsilon^{2}\right)=\eta_{1} \partial_{1} y\left(m_{1, \ell}\right)+\mathcal{O}\left(\varepsilon^{2}\right),
\end{aligned}
$$

where $m_{1, \ell}$ is the midpoint of the edge $\left[x_{\ell}, x_{\ell+e_{1}}\right]$. Notice that the above relations do not depend on the signs of $\eta_{1}, \eta_{2}$. Using similar groupings and the fact that

$$
\eta_{1} \frac{1}{4}\left[\partial_{1} y\left(m_{1, \ell}\right)+\partial_{1} y\left(m_{1, \ell+e_{2}}\right)+\partial_{1} y\left(m_{1, \ell+\eta_{2} e_{2}}\right)+\partial_{1} y\left(m_{1, \ell-\left(\eta_{2}-1\right) e_{2}}\right)\right]=\eta_{1} \partial_{1} y\left(m_{K}\right)+\mathcal{O}\left(\varepsilon^{2}\right),
$$

one can deduce, by adopting the arguments of Lemma 3.1 to our case, that

$$
\Phi^{a}(y)=\varepsilon^{d} \sum_{\ell \in \mathfrak{L}} \sum_{\eta \in R} \phi_{\eta}\left(\nabla y\left(m_{K}\right) \eta\right)+\mathcal{O}\left(\varepsilon^{2}\right) .
$$

The proof is thus complete. 


\section{AnAlysis of CAUChy-BORn APPROXimations: $d=3$}

The analysis presented in the previous sections can be extended to three space dimensions. The arguments are similar, but there are certain steps that differ, so we shall focus our attention on these, and we shall only present the key points here. In particular, we shall omit arguments that are similar to those in two dimensions, unless this is necessary. Linking the continuum model to the atomistic model is based on a three-dimensional atomistic Cauchy-Born model (A-CB). As before, to derive this model we start from the continuum model and perform appropriate approximation steps. The final model has consistency error of the order $\mathcal{O}\left(\varepsilon^{2}\right)$ compared to the continuum Cauchy-Born model and the original atomistic model.

Trilinear finite elements on the lattice. Let $\boldsymbol{V}_{\varepsilon}$ be the linear space of all periodic functions on the lattice $\mathfrak{L}$ that are continuous and piecewise trilinear on $\bar{\Omega}$. More precisely, let

$$
\begin{aligned}
& \mathcal{T}:=\left\{K \subset \Omega: \quad K=\left(x_{\ell_{1}}, x_{\ell_{1}+1}\right) \times\left(x_{\ell_{2}}, x_{\ell_{2}+1}\right) \times\left(x_{\ell_{3}}, x_{\ell_{3}+1}\right), \quad x_{\ell}=\left(x_{\ell_{1}}, x_{\ell_{2}}, x_{\ell_{3}}\right) \in \Omega_{\mathrm{discr}}\right\}, \\
& \boldsymbol{V}_{\varepsilon}:=\left\{v: \Omega \rightarrow \mathbb{R}^{d}, \quad v \in C(\Omega),\left.v\right|_{K} \in \mathbb{Q}_{1}(K) \text { and } v_{\ell}=v\left(x_{\ell}\right) \quad \text { periodic with respect to } \mathfrak{L}\right\},
\end{aligned}
$$

where $\mathbb{Q}_{1}(K)$ denotes the set of all trilinear functions on $K$. As before the elements of $\boldsymbol{V}_{\varepsilon}$ can be expressed in terms of the nodal basis functions $\Psi_{\ell}=\Psi_{\ell}(x)$ as

$$
v(x)=\sum_{\ell \in \mathfrak{L}} v_{\ell} \Psi_{\ell_{1}}\left(x_{1}\right) \Psi_{\ell_{2}}\left(x_{2}\right) \Psi_{\ell_{3}}\left(x_{3}\right), \quad v_{\ell}=v\left(x_{\ell}\right),
$$

where we have used the fact that $\Psi_{\ell}(x)$ can be written as the tensor product of the standard one-dimensional piecewise linear hat functions $\Psi_{\ell_{i}}\left(x_{i}\right)$. Here $\Psi_{\ell_{i}}\left(x_{\tilde{\ell}_{i}}\right)=\delta_{\ell_{i} \tilde{\ell}_{i}}$.

5.1. Consistency analysis of the Cauchy-Born model: $d=3$. Let, as before, $v \in \boldsymbol{V}_{\varepsilon}$ and let $y$ be a sufficiently smooth function. Our aim is to approximate

$$
\left\langle D \Phi^{C B}(y), v\right\rangle=\int_{\Omega} S_{i \alpha}(\nabla y(x)) \partial_{\alpha} v^{i}(x) \mathrm{d} x, \quad v \in \boldsymbol{V}_{\varepsilon}
$$

As before, we consider a generic term of the above sum of the form:

$$
\int_{\Omega} g(\nabla y(x)) \partial_{1} v(x) \mathrm{d} x, \quad v \in \boldsymbol{V}_{\varepsilon} .
$$

Recall that, since $v \in \boldsymbol{V}_{\varepsilon}$,

$$
\begin{aligned}
\partial_{1} v(x) & =\sum_{\ell \in \mathfrak{L}} v_{\left(\ell_{1}, \ell_{2}, \ell_{3}\right)} \frac{1}{\varepsilon}\left(1_{\left[x_{\ell_{1}-1}, x_{\ell_{1}}\right]}\left(x_{1}\right)-1_{\left[x_{\ell_{1}}, x_{\ell_{1}+1}\right]}\left(x_{1}\right)\right) \Psi_{\ell_{2}}\left(x_{2}\right) \Psi_{\ell_{3}}\left(x_{3}\right) \\
& =\sum_{\ell \in \mathfrak{L}} \frac{1}{\varepsilon}\left(v_{\left(\ell_{1}+1, \ell_{2}, \ell_{3}\right)}-v_{\left(\ell_{1}, \ell_{2}, \ell_{3}\right)}\right) 1_{\left[x_{\ell_{1}}, x_{\ell_{1}+1}\right]}\left(x_{1}\right) \Psi_{\ell_{2}}\left(x_{2}\right) \Psi_{\ell_{3}}\left(x_{3}\right) .
\end{aligned}
$$

Let us now consider

$$
G(x):=G\left(x_{1}, x_{2}, x_{3}\right)=\int_{x_{-N_{1}-1}}^{x_{1}} g\left(\nabla y\left(x_{1}^{\prime}, x_{2}, x_{3}\right)\right) \mathrm{d} x_{1}^{\prime} .
$$


Then,

$$
\begin{aligned}
& \int_{\Omega} g(\nabla y(x)) \partial_{1} v(x) \mathrm{d} x \\
& =\frac{1}{\varepsilon} \sum_{\ell \in \mathfrak{L}} \int_{\Omega} g(\nabla y(x))\left(v_{\left(\ell_{1}+1, \ell_{2}, \ell_{3}\right)}-v_{\left(\ell_{1}, \ell_{2}, \ell_{3}\right)}\right) 1_{\left[x_{\ell_{1}}, x_{\ell_{1}+1}\right]}\left(x_{1}\right) \Psi_{\ell_{2}}\left(x_{2}\right) \Psi_{\ell_{3}}\left(x_{3}\right) \mathrm{d} x \\
& =\frac{1}{\varepsilon} \sum_{\ell \in \mathfrak{L}} \int_{x_{\ell_{2}-1}}^{x_{\ell_{2}+1}} \int_{x_{\ell_{3}-1}}^{x_{\ell_{3}+1}}\left\{\int_{x_{\ell_{1}}}^{x_{\ell_{1}+1}} g(\nabla y(x)) \mathrm{d} x_{1}\left[v_{\left(\ell_{1}+1, \ell_{2}, \ell_{3}\right)}-v_{\left(\ell_{1}, \ell_{2}, \ell_{3}\right)}\right] \Psi_{\ell_{2}}\left(x_{2}\right) \Psi_{\ell_{3}}\left(x_{3}\right)\right\} \mathrm{d} x_{2} \mathrm{~d} x_{3} \\
& =\frac{1}{\varepsilon} \sum_{\ell \in \mathfrak{L}} \int_{x_{\ell_{2}-1}}^{x_{\ell_{2}+1}} \int_{x_{\ell_{3}-1}}^{x_{\ell_{3}+1}}\left[G\left(x_{\ell_{1}+1}, x_{2}, x_{3}\right)-G\left(x_{\ell_{1}}, x_{2}, x_{3}\right)\right] \Psi_{\ell_{2}}\left(x_{2}\right) \Psi_{\ell_{3}}\left(x_{3}\right) \mathrm{d} x_{2} \mathrm{~d} x_{3} \\
& =\frac{1}{\varepsilon} \sum_{\ell \in \mathfrak{L}} \int_{x_{\ell_{2}-1}}^{x_{\ell_{2}+1}} \int_{x_{\ell_{3}-1}}^{x_{\ell_{3}+1}}\left[G\left(x_{\ell_{1}+1}, x_{2}, x_{3}\right)-G\left(x_{\ell_{1}}, x_{2}, x_{3}\right)\right]\left(\Psi_{\ell_{2}}\left(x_{2}\right) \Psi_{\ell_{3}}\left(x_{3}\right)-\frac{1}{4}\right) \mathrm{d} x_{2} \mathrm{~d} x_{3} \\
& \quad \times\left[v_{\left(\ell_{1}+1, \ell_{2}, \ell_{3}\right)}-v_{\left(\ell_{1}, \ell_{2}, \ell_{3}\right)}\right] \\
& +\frac{1}{\varepsilon} \sum_{\ell \in \mathfrak{L}} \int_{x_{\ell_{2}-1}}^{x_{\ell_{2}+1}} \int_{x_{\ell_{3}-1}}^{x_{\ell_{3}+1}}\left[G\left(x_{\ell_{1}+1}, x_{2}, x_{3}\right)-G\left(x_{\ell_{1}}, x_{2}, x_{3}\right)\right] \frac{1}{4} \mathrm{~d} x_{2} \mathrm{~d} x_{3}\left[v_{\left(\ell_{1}+1, \ell_{2}, \ell_{3}\right)}-v_{\left(\ell_{1}, \ell_{2}, \ell_{3}\right)}\right] \\
& =
\end{aligned}
$$

As in two space dimensions (cf. Lemma 2.2) one can show that $A_{1}$ is second order accurate with respect to $\varepsilon$. The term $A_{2}$, on the other hand, requires further simplification. To this end, notice that

$$
\begin{aligned}
A_{2}= & \frac{1}{\varepsilon} \sum_{\ell \in \mathfrak{L}} \int_{x_{\ell_{2}-1}}^{x_{\ell_{2}+1}} \int_{x_{\ell_{3}-1}}^{x_{\ell_{3}+1}}\left[G\left(x_{\ell_{1}+1}, x_{2}, x_{3}\right)-G\left(x_{\ell_{1}}, x_{2}, x_{3}\right)\right] \frac{1}{4} \mathrm{~d} x_{2}\left[v_{\left(\ell_{1}+1, \ell_{2}, \ell_{3}\right)}-v_{\left(\ell_{1}, \ell_{2}, \ell_{3}\right)}\right] \\
= & \frac{1}{\varepsilon} \sum_{\ell \in \mathfrak{L}} \frac{1}{4} \int_{x_{\ell_{2}-1}}^{x_{\ell_{2}+1}} \int_{x_{\ell_{3}-1}}^{x_{\ell_{3}+1}}\left\{\int_{x_{\ell_{1}}}^{x_{\ell_{1}+1}} g(\nabla y(x)) \mathrm{d} x_{1}\right\} \mathrm{d} x_{2}\left[v_{\left(\ell_{1}+1, \ell_{2}, \ell_{3}\right)}-v_{\left(\ell_{1}, \ell_{2}, \ell_{3}\right)}\right] \\
= & \frac{1}{\varepsilon} \sum_{\ell \in \mathfrak{L}} \frac{1}{4} \int_{x_{\ell_{2}}}^{x_{\ell_{2}+1}} \int_{x_{\ell_{3}}}^{x_{\ell_{3}+1}}\left\{\int_{x_{\ell_{1}}}^{x_{\ell_{1}+1}} g(\nabla y(x)) \mathrm{d} x_{1}\right\} \mathrm{d} x_{2}\left[v_{\left(\ell_{1}+1, \ell_{2}, \ell_{3}\right)}-v_{\left(\ell_{1}, \ell_{2}, \ell_{3}\right)}\right] \\
& +\frac{1}{\varepsilon} \sum_{\tilde{\ell} \in \mathfrak{L}} \frac{1}{4} \int_{x_{\ell_{2}}}^{x_{\ell_{2}+1}} \int_{x_{\tilde{\ell}_{3}}}^{x_{\tilde{\ell}_{3}+1}}\left\{\int_{x_{\ell_{1}}}^{x_{\ell_{1}+1}} g(\nabla y(x)) \mathrm{d} x_{1}\right\} \mathrm{d} x_{2}\left[v_{\left(\ell_{1}+1, \ell_{2}, \tilde{\ell}_{3}+1\right)}-v_{\left(\ell_{1}, \ell_{2}, \tilde{\ell}_{3}+1\right)}\right] \\
& +\frac{1}{\varepsilon} \sum_{\ell^{\prime} \in \mathfrak{L}} \frac{1}{4} \int_{x_{\ell_{2}^{\prime}}}^{x_{\ell_{2}^{\prime}+1}} \int_{x_{\ell_{3}}}^{x_{\ell_{3}+1}}\left\{\int_{x_{\ell_{1}}}^{x_{\ell_{1}+1}} g(\nabla y(x)) \mathrm{d} x_{1}\right\} \mathrm{d} x_{2}\left[v_{\left.\left(\ell_{1}+1, \ell_{2}^{\prime}+1, \ell_{3}\right)\right)}-v_{\left.\left(\ell_{1}, \ell_{2}^{\prime}+1, \ell_{3}\right)\right)}\right] \\
& +\frac{1}{\varepsilon} \sum_{\hat{\ell} \in \mathfrak{L}} \frac{1}{4} \int_{x_{\ell_{2}^{\prime}}}^{x_{\ell_{2}^{\prime}+1}} \int_{x_{\tilde{\ell}_{3}}}^{x_{\tilde{\ell}_{3}+1}}\left\{\int_{x_{\ell_{1}}}^{x_{\ell_{1}+1}} g(\nabla y(x)) \mathrm{d} x_{1}\right\} \mathrm{d} x_{2}\left[v_{\left(\ell_{1}+1, \ell_{2}^{\prime}+1, \tilde{\ell}_{3}+1\right)}-v_{\left(\ell_{1}, \ell_{2}^{\prime}+1, \tilde{\ell}_{3}+1\right)}\right] \\
= & \sum_{K \in \mathcal{T}} \int_{K} g(\nabla y(x)) \mathrm{d} x \frac{1}{4}\left\{\bar{D}_{e_{1}} v_{\ell}+\bar{D}_{e_{1}} v_{\ell+e_{2}}+\bar{D}_{e_{1}} v_{\ell+e_{3}}+\bar{D}_{e_{1}} v_{\ell+e_{2}+e_{3}}\right\},
\end{aligned}
$$


where $\tilde{\ell}=\left(\ell_{1}, \ell_{2}, \tilde{\ell}_{3}\right), \ell^{\prime}=\left(\ell_{1}, \ell_{2}^{\prime}, \ell_{3}\right)$, and $\hat{\ell}=\left(\ell_{1}, \ell_{2}^{\prime}, \tilde{\ell}_{3}\right)$. We define the average discrete derivatives as follows:

$$
\overline{\bar{D}}_{e_{1}} v_{\ell}=\frac{1}{4}\left\{\bar{D}_{e_{1}} v_{\ell}+\bar{D}_{e_{1}} v_{\ell+e_{2}}+\bar{D}_{e_{1}} v_{\ell+e_{3}}+\bar{D}_{e_{1}} v_{\ell+e_{2}+e_{3}}\right\}
$$

As in Lemma 2.3, one can show, with $m_{K}$ signifying the barycenter of $K$, that there exists a constant $C=C(y)$ such that

$$
\left|\sum_{K \in \mathcal{T}}\left[\int_{K} g(\nabla y(x)) \mathrm{d} x-|K| g\left(\nabla y\left(m_{K}\right)\right)\right] \overline{\bar{D}}_{e_{1}} v_{\ell}\right| \leq C(y) \varepsilon^{2}|v|_{W^{1, p}(\Omega)} .
$$

As in two space dimensions, one can define, for any $v \in \boldsymbol{V}_{\varepsilon}$, the quantity

$$
\begin{aligned}
\left\langle\mathcal{A}^{a, C B}, v\right\rangle_{\varepsilon}:= & \varepsilon^{d} \sum_{K \in \mathcal{T}} \sum_{\eta \in R}\left\{\nabla_{\zeta} \phi_{\eta}\left(\nabla y\left(m_{K}\right) \eta\right) \eta_{1}\right\} \cdot \overline{\bar{D}}_{e_{1}} v_{\ell} \\
& +\varepsilon^{d} \sum_{K \in \mathcal{T}} \sum_{\eta \in R}\left\{\nabla_{\zeta} \phi_{\eta}\left(\nabla y\left(m_{K}\right) \eta\right) \eta_{2}\right\} \cdot \overline{\bar{D}}_{e_{2}} v_{\ell} \\
& +\varepsilon^{d} \sum_{K \in \mathcal{T}} \sum_{\eta \in R}\left\{\nabla_{\zeta} \phi_{\eta}\left(\nabla y\left(m_{K}\right) \eta\right) \eta_{3}\right\} \cdot \overline{\bar{D}}_{e_{3}} v_{\ell} .
\end{aligned}
$$

We thus deduce that $\left\langle\mathcal{A}^{a, C B}, v\right\rangle_{\varepsilon}$ is a second order approximation to $\left\langle D \Phi^{C B}(y), v\right\rangle$ in the sense that

$$
\left|\left\langle D \Phi^{C B}(y), v\right\rangle-\left\langle\mathcal{A}^{a, C B}, v\right\rangle_{\varepsilon}\right| \leq M \varepsilon^{2}|v|_{W^{1, p}(\Omega)} .
$$

5.2. The atomistic Cauchy-Born model: $d=3$. Using the above definition of the average discrete derivatives we define the discrete gradient matrix as

$$
\left\{\bar{\nabla} v_{\ell}\right\}_{i \alpha}:=\overline{\bar{D}}_{e_{\alpha}} v_{\ell}^{i}
$$

and the atomistic potential

$$
\Phi^{a, C B}(y):=\varepsilon^{d} \sum_{\ell \in \mathfrak{L}} \sum_{\eta \in R} \phi_{\eta}\left(\bar{\nabla} y_{\ell} \eta\right)=\varepsilon^{d} \sum_{\ell \in \mathfrak{L}} W_{C B}\left(\bar{\nabla} y_{\ell}\right) .
$$

Now, for a given field of external forces $f: \mathfrak{L} \rightarrow \mathbb{R}^{d}$ the atomistic Cauchy-Born problem reads as follows:

$$
\begin{aligned}
& \text { find a local minimizer } y^{a, C B} \text { in } \mathcal{X} \text { of : } \\
& \Phi^{a, C B}\left(y^{a, C B}\right)-\left\langle f, y^{a}\right\rangle_{\varepsilon} .
\end{aligned}
$$

If such a minimizer exists, then

$$
\left\langle D \Phi^{a, C B}\left(y^{a, C B}\right), v\right\rangle_{\varepsilon}=\langle f, v\rangle_{\varepsilon}, \quad \text { for all } v \in \mathscr{V} .
$$

As in two space dimensions, one can link $\mathcal{A}^{a, C B}$ to $D \Phi^{a, C B}$ as follows: let $y \in \boldsymbol{V}_{\varepsilon}$; then, for any $v \in \boldsymbol{V}_{\varepsilon}$,

$$
\left\langle\mathcal{A}^{a, C B}, v\right\rangle_{\varepsilon}=\left\langle D \Phi^{a, C B}(y), v\right\rangle_{\varepsilon} .
$$


5.3. Comparison of atomistic Cauchy-Born and atomistic models: $d=3$. To compare the atomistic and atomistic Cauchy-Born models we start from (1.4):

$$
\left\langle D \Phi^{a}(y), v\right\rangle_{\varepsilon}=\varepsilon^{d} \sum_{\ell \in \mathfrak{L}} \sum_{\eta \in R} \nabla_{\zeta} \phi_{\eta}\left(\bar{D}_{\eta} y_{\ell}\right) \cdot \bar{D}_{\eta} v_{\ell}
$$

Then, one can split $\bar{D}_{\eta} v_{\ell}$ and $\bar{D}_{\eta} v_{\ell}$ as follows:

$$
\bar{D}_{\eta} v_{\ell}=\bar{D}_{\eta_{1} e_{1}} v_{\ell}+\bar{D}_{\eta_{2} e_{2}} v_{\ell+\eta_{1} e_{1}}+\bar{D}_{\eta_{3} e_{3}} v_{\ell+\eta_{1} e_{1}+\eta_{2} e_{2}}
$$

and

$$
\bar{D}_{\eta} v_{\ell}=\bar{D}_{\eta_{1} e_{1}} v_{\ell}+\bar{D}_{\eta_{3} e_{3}} v_{\ell+\eta_{1} e_{1}}+\bar{D}_{\eta_{2} e_{2}} v_{\ell+\eta_{1} e_{1}+\eta_{3} e_{3}} .
$$

Using similar splittings but starting with $\bar{D}_{\eta_{2} e_{2}} v_{\ell}$ and $\bar{D}_{\eta_{3} e_{3}} v_{\ell}$, we end up with six alternative expressions for $\bar{D}_{\eta} v_{\ell}$. Thus, by replacing $\bar{D}_{\eta} v_{\ell}$ with its average and grouping terms of the same type of discrete derivative we deduce that

$$
\begin{aligned}
& \left\langle D \Phi^{a}(y), v\right\rangle_{\varepsilon} \\
& =\varepsilon^{d} \sum_{\ell \in \mathfrak{L}} \sum_{\eta \in R} \nabla_{\zeta} \phi_{\eta}\left(\bar{D}_{\eta} y_{\ell}\right) \cdot\left\{\frac{1}{3} \bar{D}_{\eta_{1} e_{1}} v_{\ell}+\frac{1}{6} \bar{D}_{\eta_{1} e_{1}} v_{\ell+\eta_{2} e_{2}}+\frac{1}{6} \bar{D}_{\eta_{1} e_{1}} v_{\ell+\eta_{3} e_{3}}+\frac{1}{3} \bar{D}_{\eta_{1} e_{1}} v_{\ell+\eta_{2} e_{2}+\eta_{3} e_{3}}\right\} \\
& +\varepsilon^{d} \sum_{\ell \in \mathfrak{L}} \sum_{\eta \in R} \nabla_{\zeta} \phi_{\eta}\left(\bar{D}_{\eta} y_{\ell}\right) \cdot\left\{\frac{1}{3} \bar{D}_{\eta_{2} e_{2}} v_{\ell}+\frac{1}{6} \bar{D}_{\eta_{2} e_{2}} v_{\ell+\eta_{1} e_{1}}+\frac{1}{6} \bar{D}_{\eta_{2} e_{2}} v_{\ell+\eta_{3} e_{3}}+\frac{1}{3} \bar{D}_{\eta_{2} e_{2}} v_{\ell+\eta_{1} e_{1}+\eta_{3} e_{3}}\right\} \\
& +\varepsilon^{d} \sum_{\ell \in \mathfrak{L}} \sum_{\eta \in R} \nabla_{\zeta} \phi_{\eta}\left(\bar{D}_{\eta} y_{\ell}\right) \cdot\left\{\frac{1}{3} \bar{D}_{\eta_{3} e_{3}} v_{\ell}+\frac{1}{6} \bar{D}_{\eta_{3} e_{3}} v_{\ell+\eta_{1} e_{1}}+\frac{1}{6} \bar{D}_{\eta_{3} e_{3}} v_{\ell+\eta_{2} e_{2}}+\frac{1}{3} \bar{D}_{\eta_{3} e_{3}} v_{\ell+\eta_{1} e_{1}+\eta_{2} e_{2}}\right\} .
\end{aligned}
$$

Hence, since the lattice is periodic, we deduce that

$$
\begin{aligned}
& \left\langle D \Phi^{a}(y), v\right\rangle_{\varepsilon} \\
& =\varepsilon^{d} \sum_{\ell \in \mathfrak{L}} \sum_{\eta \in R}\left\{\frac{1}{3} \nabla_{\zeta} \phi_{\eta}\left(\bar{D}_{\eta} y_{\ell}\right)+\frac{1}{6} \nabla_{\zeta} \phi_{\eta}\left(\bar{D}_{\eta} y_{\ell-\eta_{2} e_{2}}\right)+\frac{1}{6} \nabla_{\zeta} \phi_{\eta}\left(\bar{D}_{\eta} y_{\ell-\eta_{3} e_{3}}\right)+\frac{1}{3} \nabla_{\zeta} \phi_{\eta}\left(\bar{D}_{\eta} y_{\ell-\eta_{2} e_{2}-\eta_{3} e_{3}}\right)\right\} \\
& +\varepsilon^{d} \sum_{\ell \in \mathfrak{L}} \sum_{\eta \in R}\left\{\frac{1}{3} \nabla_{\zeta} \phi_{\eta}\left(\bar{D}_{\eta} y_{\ell}\right)+\frac{1}{6} \nabla_{\zeta} \phi_{\eta}\left(\bar{D}_{\eta} y_{\ell-\eta_{1} e_{1}}\right)+\frac{1}{6} \nabla_{\zeta} \phi_{\eta}\left(\bar{D}_{\eta} y_{\ell-\eta_{3} e_{3}}\right)+\frac{1}{3} \nabla_{\zeta} \phi_{\eta}\left(\bar{D}_{\eta} y_{\ell-\eta_{1} e_{1}-\eta_{3} e_{3}}\right)\right\} \\
& +\varepsilon^{d} \sum_{\ell \in \mathfrak{L}} \sum_{\eta \in R}\left\{\frac{1}{3} \nabla_{\zeta} \phi_{\eta}\left(\bar{D}_{\eta} y_{\ell}\right)+\frac{1}{6} \nabla_{\zeta} \phi_{\eta}\left(\bar{D}_{\eta} y_{\ell-\eta_{1} e_{1}}\right)+\frac{1}{6} \nabla_{\zeta} \phi_{\eta}\left(\bar{D}_{\eta} y_{\ell-\eta_{2} e_{2}}\right)+\frac{1}{3} \nabla_{\zeta} \phi_{\eta}\left(\bar{D}_{\eta} y_{\ell-\eta_{1} e_{1}-\eta_{2} e_{2}}\right)\right\} \\
& \cdot \bar{D}_{\eta_{3} e_{3} v_{\ell} .}
\end{aligned}
$$

As before we split, for $\eta_{1}, \eta_{2}, \eta_{3}>0$,

$$
\begin{aligned}
& \bar{D}_{\eta_{1} e_{1}} v_{\ell}=\bar{D}_{e_{1}} v_{\ell}+\cdots+\bar{D}_{e_{1}} v_{\ell+\left(\eta_{1}-1\right) e_{1}}, \\
& \bar{D}_{\eta_{2} e_{2}} v_{\ell}=\bar{D}_{e_{2}} v_{\ell}+\cdots+\bar{D}_{e_{2}} v_{\ell+\left(\eta_{2}-1\right) e_{2}}, \\
& \bar{D}_{\eta_{3} e_{3}} v_{\ell}=\bar{D}_{e_{3}} v_{\ell}+\cdots+\bar{D}_{e_{3}} v_{\ell+\left(\eta_{3}-1\right) e_{3}}
\end{aligned}
$$

In case where $\eta_{\alpha}<0$ we use splittings of the form (3.2). In the sequel we will assume that $\eta_{1}, \eta_{2}, \eta_{3}>0$. The general case can be treated by adopting the arguments presented in two space dimensions (cf. (3.3) and 
Lemma 3.2). Therefore, by using the notation

$$
\begin{aligned}
& \bar{\Phi}_{\eta, \ell, 1}:=\sum_{k=0}^{\eta_{1}-1}\left\{\frac{1}{3} \nabla_{\zeta} \phi_{\eta}\left(\bar{D}_{\eta} y_{\ell-k e_{1}}\right)+\frac{1}{6} \nabla_{\zeta} \phi_{\eta}\left(\bar{D}_{\eta} y_{\ell-k e_{1}-\eta_{2} e_{2}}\right)\right. \\
& \left.+\frac{1}{6} \nabla_{\zeta} \phi_{\eta}\left(\bar{D}_{\eta} y_{\ell-k e_{1}-\eta_{3} e_{3}}\right)+\frac{1}{3} \nabla_{\zeta} \phi_{\eta}\left(\bar{D}_{\eta} y_{\ell-k e_{1}-\eta_{2} e_{2}-\eta_{3} e_{3}}\right)\right\}, \\
& \bar{\Phi}_{\eta, \ell, 2}:=\sum_{k=0}^{\eta_{2}-1}\left\{\frac{1}{3} \nabla_{\zeta} \phi_{\eta}\left(\bar{D}_{\eta} y_{\ell-k e_{2}}\right)+\frac{1}{6} \nabla_{\zeta} \phi_{\eta}\left(\bar{D}_{\eta} y_{\ell-k e_{2}-\eta_{1} e_{1}}\right)\right. \\
& \left.+\frac{1}{6} \nabla_{\zeta} \phi_{\eta}\left(\bar{D}_{\eta} y_{\ell-k e_{2}-\eta_{3} e_{3}}\right)+\frac{1}{3} \nabla_{\zeta} \phi_{\eta}\left(\bar{D}_{\eta} y_{\ell-k e_{2}-\eta_{1} e_{1}-\eta_{3} e_{3}}\right)\right\}, \\
& \bar{\Phi}_{\eta, \ell, 3}:=\sum_{k=0}^{\eta_{3}-1}\left\{\frac{1}{3} \nabla_{\zeta} \phi_{\eta}\left(\bar{D}_{\eta} y_{\ell-k e_{3}}\right)+\frac{1}{6} \nabla_{\zeta} \phi_{\eta}\left(\bar{D}_{\eta} y_{\ell-k e_{3}-\eta_{1} e_{1}}\right)\right. \\
& \left.+\frac{1}{6} \nabla_{\zeta} \phi_{\eta}\left(\bar{D}_{\eta} y_{\ell-k e_{3}-\eta_{2} e_{2}}\right)+\frac{1}{3} \nabla_{\zeta} \phi_{\eta}\left(\bar{D}_{\eta} y_{\ell-k e_{3}-\eta_{1} e_{1}-\eta_{2} e_{2}}\right)\right\},
\end{aligned}
$$

we finally deduce that

$$
\begin{aligned}
\left\langle D \Phi^{a}(y), v\right\rangle_{\varepsilon}= & \varepsilon^{d} \sum_{\ell \in \mathfrak{L}} \sum_{\eta \in R} \bar{\Phi}_{\eta, \ell, 1} \cdot \bar{D}_{e_{1}} v_{\ell} \\
& +\varepsilon^{d} \sum_{\ell \in \mathfrak{L}} \sum_{\eta \in R} \bar{\Phi}_{\eta, \ell, 2} \cdot \bar{D}_{e_{2}} v_{\ell}+\varepsilon^{d} \sum_{\ell \in \mathfrak{L}} \sum_{\eta \in R} \bar{\Phi}_{\eta, \ell, 3} \cdot \bar{D}_{e_{3}} v_{\ell} .
\end{aligned}
$$

Next, we shall focus on the comparison of $\bar{\Phi}_{\eta, \ell, 1}$ with the term

$$
\Phi_{\eta, \ell, 1}=\sum_{k=0}^{\eta_{1}-1} \nabla_{\zeta} \phi_{\eta}\left(\nabla y\left(m_{k, \eta_{1}, \ell}\right) \eta\right),
$$

where

$$
\begin{aligned}
& m_{k, \eta_{1}, \ell} \quad \text { is the midpoint of the side with endpoints } x_{\ell-k e_{1}}, x_{\ell-k e_{1}+\eta_{1} e_{1}} \text {, and } \\
& m_{1, \ell} \quad \text { is the midpoint of the side with endpoints } x_{\ell}, x_{\ell+e_{1}} \text {. }
\end{aligned}
$$

Let $k=0$. We group together the $\frac{1}{3}$-terms in $\bar{\Phi}_{\eta, \ell, 1}$. We use the splittings

$$
\begin{aligned}
\bar{D}_{\eta} v_{\ell}=\frac{1}{2}\{ & \bar{D}_{\eta_{2} e_{2}} v_{\ell}+\bar{D}_{\eta_{3} e_{3}} v_{\ell+\eta_{2} e_{2}}+\bar{D}_{\eta_{1} e_{1}} v_{\ell+\eta_{2} e_{2}+\eta_{3} e_{3}} \\
& \left.+\bar{D}_{\eta_{1} e_{1}} v_{\ell}+\bar{D}_{\eta_{3} e_{3}} v_{\ell+\eta_{1} e_{1}}+\bar{D}_{\eta_{2} e_{2}} v_{\ell+\eta_{1} e_{1}+\eta_{3} e_{3}}\right\}
\end{aligned}
$$

and

$$
\begin{aligned}
\bar{D}_{\eta} v_{\ell-\eta_{2} e_{2}-\eta_{3} e_{3}=}=\frac{1}{2}\{ & \bar{D}_{\eta_{2} e_{2}} v_{\ell-\eta_{2} e_{2}-\eta_{3} e_{3}}+\bar{D}_{\eta_{3} e_{3}} v_{\ell-\eta_{3} e_{3}}+\bar{D}_{\eta_{1} e_{1}} v_{\ell} \\
& \left.+\bar{D}_{\eta_{1} e_{1}} v_{\ell-\eta_{2} e_{2}-\eta_{3} e_{3}}+\bar{D}_{\eta_{3} e_{3}} v_{\ell-\eta_{2} e_{2}-\eta_{3} e_{3}+\eta_{1} e_{1}}+\bar{D}_{\eta_{2} e_{2}} v_{\ell-\eta_{2} e_{2}+\eta_{1} e_{1}}\right\} .
\end{aligned}
$$

These choices were made in order to create the required symmetries. In fact, by taking into account that $\bar{D}_{\eta} v_{\ell}$ and $\bar{D}_{\eta} v_{\ell-\eta_{2} e_{2}-\eta_{3} e_{3}}$ are grouped together in (5.5) and observing that

$$
\begin{aligned}
& 2 \bar{D}_{\eta_{1} e_{1}} v_{\ell}+\bar{D}_{\eta_{1} e_{1}} v_{\ell+\eta_{2} e_{2}+\eta_{3} e_{3}}+\bar{D}_{\eta_{1} e_{1}} v_{\ell-\eta_{2} e_{2}-\eta_{3} e_{3}}=4 \eta_{1} \partial_{1} y\left(m_{0, \eta_{1}, \ell}\right)+\mathcal{O}\left(\varepsilon^{2}\right), \\
& \bar{D}_{\eta_{2} e_{2}} v_{\ell}+\bar{D}_{\eta_{2} e_{2}} v_{\ell+\eta_{1} e_{1}+\eta_{3} e_{3}}+\bar{D}_{\eta_{2} e_{2}} v_{\ell-\eta_{2} e_{2}-\eta_{3} e_{3}}+\bar{D}_{\eta_{2} e_{2}} v_{\ell-\eta_{2} e_{2}+\eta_{1} e_{1}}=4 \eta_{2} \partial_{2} y\left(m_{0, \eta_{1}, \ell}\right)+\mathcal{O}\left(\varepsilon^{2}\right), \\
& \bar{D}_{\eta_{3} e_{3}} v_{\ell+\eta_{2} e_{2}}+\bar{D}_{\eta_{3} e_{3}} v_{\ell+\eta_{1} e_{1}}+\bar{D}_{\eta_{3} e_{3}} v_{\ell-\eta_{3} e_{3}}+\bar{D}_{\eta_{3} e_{3}} v_{\ell-\eta_{2} e_{2}-\eta_{3} e_{3}+\eta_{1} e_{1}}=4 \eta_{3} \partial_{3} y\left(m_{0, \eta_{1}, \ell}\right)+\mathcal{O}\left(\varepsilon^{2}\right),
\end{aligned}
$$


yields a second order approximation to $\frac{4}{6} \nabla_{\zeta} \phi_{\eta}\left(\nabla y\left(m_{0, \eta_{1}, \ell}\right) \eta\right)$, upon employing similar arguments as in Lemma 3.1. The remaining $\frac{2}{6}$ factor is due to the $\frac{1}{6}$-terms in $\bar{\Phi}_{\eta, \ell, 1}$. In fact, we use the splittings

$$
\begin{aligned}
\bar{D}_{\eta} v_{\ell-k e_{1}-\eta_{2} e_{2}}=\frac{1}{2}\{ & \bar{D}_{\eta_{2} e_{2}} v_{\ell-k e_{1}-\eta_{2} e_{2}}+\bar{D}_{\eta_{3} e_{3}} v_{\ell-k e_{1}}+\bar{D}_{\eta_{1} e_{1}} v_{\ell-k e_{1}+\eta_{3} e_{3}} \\
& \left.+\bar{D}_{\eta_{1} e_{1}} v_{\ell-k e_{1}-\eta_{2} e_{2}}+\bar{D}_{\eta_{3} e_{3}} v_{\ell-\eta_{2} e_{2}}+\bar{D}_{\eta_{2} e_{2}} v_{\ell-\eta_{2} e_{2}+\eta_{3} e_{3}}\right\}
\end{aligned}
$$

and

$$
\begin{aligned}
\bar{D}_{\eta} v_{\ell-k e_{1}-\eta_{3} e_{3}}=\frac{1}{2}\{ & \bar{D}_{\eta_{2} e_{2}} v_{\ell-k e_{1}-\eta_{3} e_{3}}+\bar{D}_{\eta_{3} e_{3}} v_{\ell-k e_{1}-\eta_{3} e_{3}+\eta_{2} e_{2}}+\bar{D}_{\eta_{1} e_{1}} v_{\ell-k e_{1}+\eta_{2} e_{2}} \\
& \left.+\bar{D}_{\eta_{1} e_{1}} v_{\ell-k e_{1}-\eta_{3} e_{3}}+\bar{D}_{\eta_{3} e_{3}} v_{\ell-\eta_{3} e_{3}}+\bar{D}_{\eta_{2} e_{2}} v_{\ell-\eta_{3} e_{3}+\eta_{3} e_{3}}\right\} .
\end{aligned}
$$

As before,

$$
\begin{gathered}
\bar{D}_{\eta_{1} e_{1}} v_{\ell-k e_{1}+\eta_{3} e_{3}}+\bar{D}_{\eta_{1} e_{1}} v_{\ell-k e_{1}-\eta_{2} e_{2}}+\bar{D}_{\eta_{1} e_{1}} v_{\ell-k e_{1}+\eta_{2} e_{2}}+\bar{D}_{\eta_{1} e_{1}} v_{\ell-k e_{1}-\eta_{3} e_{3}} \\
=4 \eta_{1} \partial_{1} y\left(m_{0, \eta_{1}, \ell}\right)+\mathcal{O}\left(\varepsilon^{2}\right) \\
\bar{D}_{\eta_{2} e_{2}} v_{\ell-k e_{1}-\eta_{2} e_{2}}+\bar{D}_{\eta_{2} e_{2}} v_{\ell-\eta_{2} e_{2}+\eta_{3} e_{3}}+\bar{D}_{\eta_{2} e_{2}} v_{\ell-k e_{1}-\eta_{3} e_{3}}+\bar{D}_{\eta_{2} e_{2}} v_{\ell-\eta_{3} e_{3}+\eta_{3} e_{3}} \\
=4 \eta_{2} \partial_{2} y\left(m_{0, \eta_{1}, \ell}\right)+\mathcal{O}\left(\varepsilon^{2}\right), \\
\bar{D}_{\eta_{3} e_{3}} v_{\ell-k e_{1}}+\bar{D}_{\eta_{3} e_{3}} v_{\ell-\eta_{2} e_{2}}+\bar{D}_{\eta_{3} e_{3}} v_{\ell-k e_{1}-\eta_{3} e_{3}+\eta_{2} e_{2}}+\bar{D}_{\eta_{3} e_{3}} v_{\ell-\eta_{3} e_{3}} \\
=4 \eta_{3} \partial_{3} y\left(m_{0, \eta_{1}, \ell}\right)+\mathcal{O}\left(\varepsilon^{2}\right) .
\end{gathered}
$$

We thus deduce that

$$
\left|\bar{\Phi}_{\eta, \ell, 1}-\Phi_{\eta, \ell, 1}\right| \leq C(y) \varepsilon^{2} .
$$

Finally, as in the case of two space dimensions, we have that

$$
\left|\eta_{1} \nabla_{\zeta} \phi_{\eta}\left(\nabla y\left(m_{1, \ell}\right) \eta\right)-\Phi_{\eta, \ell, 1}\right| \leq C(y) \varepsilon^{2},
$$

with $m_{1, \ell}$ signifying the midpoint of the side with endpoints $x_{\ell}, x_{\ell+e_{1}}$. By employing entirely similar arguments as in the case of two space dimensions, we then deduce that Theorem 4.1 holds in three space dimensions as well. Analogously, Theorem 4.2 is also valid in three dimensions.

\section{REMARKS ON CONVERGENCE}

We briefly discuss here how the consistency results presented in this paper, combined with appropriate local stability properties of the Cauchy-Born solution, can imply local convergence. In one space dimension these results are based on a simple application of the inverse function theorem, see [36], [31], [33], see also [24]. The stability in multiple space dimensions is very subtle (see, for example, [24]) and is beyond the scope of this paper. A key assumption in the inverse function theorem in the form presented in [12, Theorem 2.1] is that $G(w)=D \Phi^{a}(w)$ is differentiable with bounded inverse at a given point $w \in X$. Next, for the sake of simplicity of the presentation, we shall assume that in an appropriate discrete space $X_{\varepsilon}, D \Phi^{a}\left(y^{C B}\right)$ evaluated on a sufficiently smooth solution $y^{C B}$ of (1.6), whose existence is assumed, is differentiable with bounded inverse. Then, upon verifying two further assumptions on $D \Phi^{a}$ and $y^{C B}$ one can apply the inverse function theorem to infer the local existence of a solution of the atomistic problem $y^{a}$ in a ball with center $y^{C B}$. Hence, in addition, one obtains an estimate of the form

$$
\left\|y^{a}-y^{C B}\right\|_{X_{\varepsilon}} \leq \gamma\left\|D \Phi^{a}\left(y^{C B}\right)-f\right\|_{Y_{\varepsilon}} .
$$


Here $\gamma$ depends on the norm of the inverse of the derivative of $D \Phi^{a}\left(y^{C B}\right)$ and

$$
\left\|D \Phi^{a}\left(y^{C B}\right)-f\right\|_{Y_{\varepsilon}}=\sup \left\{\frac{\left|\left\langle D \Phi^{a}\left(y^{C B}\right)-f, v\right\rangle_{\varepsilon}\right|}{\|v\|_{X_{\varepsilon}}}: \quad v \in X_{\varepsilon} \quad \text { with }\|v\|_{X_{\varepsilon}} \neq 0\right\} .
$$

Thus, to estimate the error we observe that

$$
\begin{aligned}
\left\langle D \Phi^{a}\left(y^{C B}\right), v\right\rangle_{\varepsilon}-\langle f, v\rangle_{\varepsilon} & =\left[\left\langle D \Phi^{a}\left(y^{C B}\right), v\right\rangle_{\varepsilon}-\left\langle D \Phi^{C B}\left(y^{C B}\right), v\right\rangle\right]+\left[\langle f, v\rangle-\langle f, v\rangle_{\varepsilon}\right] \\
& =: I_{1}+I_{2} .
\end{aligned}
$$

Let us also suppose that $X_{\varepsilon}$ is a subspace of $\boldsymbol{V}_{\varepsilon}$ equipped with $W^{1, p}(\Omega)$ norm. Then, the consistency result of Theorem 4.1 implies that

$$
\left|I_{1}\right| \leq C\left(y^{C B}\right) \varepsilon^{2}|v|_{W^{1, p}(\Omega)} \leq C\left(y^{C B}\right) \varepsilon^{2}\|v\|_{X_{\varepsilon}} .
$$

In addition,

$$
\begin{aligned}
I_{2} & =\langle f, v\rangle-\langle f, v\rangle_{\varepsilon}=\int_{\Omega}\left(f v-Q_{I}(f v)\right) \mathrm{d} x \\
& =\sum_{K \in \mathcal{T}} \int_{K}\left(f v-Q_{I}(f v)\right) \mathrm{d} x,
\end{aligned}
$$

where $Q_{I}$ denotes the standard nodal interpolation operator on $\boldsymbol{V}_{\varepsilon}$. Let us denote by $\zeta$ the functional

$$
\zeta(w)=\frac{1}{|K|} \int_{K}\left\{w-Q_{I}(w)\right\} \mathrm{d} x, \quad w \in C(\bar{K}),
$$

and observe that $\zeta(\varphi)=0$ for all $\varphi \in \mathbb{Q}_{1}(K)$. Lemma 2.1 and inequality (2.1) then yield that

$$
|\zeta(w)| \leq C \varepsilon^{2} \sum_{\alpha=1}^{d}\left\|\partial_{\alpha}^{2} w\right\|_{L^{\infty}(K)} .
$$

Further, since $v \in \boldsymbol{V}_{\varepsilon}$, we have that $\left\|\partial_{\alpha}^{2}(f v)\right\|_{L^{\infty}(K)} \leq 3\|f\|_{W^{2, \infty}(K)}\|v\|_{W^{1, \infty}(K)}$, and we deduce, using similar arguments as in the proof of Lemma 2.3, that

$$
\left|I_{2}\right| \leq C\|f\|_{W^{2, \infty}(\Omega)} \varepsilon^{2}|v|_{W^{1, p}(\Omega)} \leq C \varepsilon^{2}\|v\|_{X_{\varepsilon}} .
$$

We thus arrive at the error bound

$$
\left\|y^{a}-y^{C B}\right\|_{X_{\varepsilon}} \leq C\left(y^{C B}, f\right) \varepsilon^{2},
$$

where $\left\|y^{a}-y^{C B}\right\|_{X_{\varepsilon}}$ is a discrete $W^{1, p}(\Omega)$ norm corresponding to $\left\|y^{a}-Q_{I} y^{C B}\right\|_{W^{1, p}(\Omega)}$.

Acknowledgements. CM was partially supported by the FP7-REGPOT project ACMAC: Archimedes Center for Modeling, Analysis and Computations of the University of Crete. ES was partially supported by the EPSRC Science and Innovation award to the Oxford Centre for Nonlinear PDE (EP/E035027/1).

\section{REFERENCES}

[1] A. Abdulle, P. Lin, and A. V. Shapeev. Numerical methods for multilattices. Technical report, arXiv:1107.3462, 2011.

[2] M. Arndt and M. Griebel. Derivation of higher order gradient continuum models from atomistic models for crystalline solids. Multiscale Model. Simul., 4(2):531-562 (electronic), 2005.

[3] M. Arndt and M. Luskin. Error estimation and atomistic-continuum adaptivity for the quasicontinuum approximation of a Frenkel-Kontorova model. Multiscale Model. Simul., 7(1):147-170, 2008.

[4] M. Arndt and M. Luskin. Goal-oriented adaptive mesh refinement for the quasicontinuum approximation of a FrenkelKontorova model. Comput. Methods Appl. Mech. Engrg., 197(49-50):4298-4306, 2008. 
[5] S. Badia, P. Bochev, M. Gunzburger, R. Lehoucq, and M. Parks. Bridging methods for coupling atomistic and continuum models. In Large-scale scientific computing, volume 4818 of Lecture Notes in Comput. Sci., pages 16-27. Springer, Berlin, 2008.

[6] S. Badia, M. Parks, P. Bochev, M. Gunzburger, and R. Lehoucq. On atomistic-to-continuum coupling by blending. Multiscale Model. Simul., 7(1):381-406, 2008.

[7] X. Blanc, C. Le Bris, and F. Legoll. Analysis of a prototypical multiscale method coupling atomistic and continuum mechanics. M2AN Math. Model. Numer. Anal., 39(4):797-826, 2005.

[8] X. Blanc, C. Le Bris, and F. Legoll. Analysis of a prototypical multiscale method coupling atomistic and continuum mechanics: the convex case. Acta Math. Appl. Sin. Engl. Ser., 23(2):209-216, 2007.

[9] X. Blanc, C. Le Bris, and P.-L. Lions. From molecular models to continuum mechanics. Arch. Ration. Mech. Anal., 164(4):341-381, 2002.

[10] X. Blanc, C. Le Bris, and P.-L. Lions. Atomistic to continuum limits for computational materials science. M2AN Math. Model. Numer. Anal., 41(2):391-426, 2007.

[11] S. C. Brenner and R. Scott. The Mathematical Theory of Finite Element Methods (Texts in Applied Mathematics). Springer, 1994.

[12] G. Caloz and J. Rappaz. Numerical analysis for nonlinear and bifurcation problems. In Handbook of numerical analysis, Vol. $V$, Handb. Numer. Anal., V, pages 487-637. North-Holland, Amsterdam, 1997.

[13] P. Ciarlet. The finite element method for elliptic problems. North-Holland, 1978.

[14] M. Dobson and M. Luskin. Analysis of a force-based quasicontinuum approximation. M2AN Math. Model. Numer. Anal., 42(1):113-139, 2008.

[15] M. Dobson and M. Luskin. Iterative solution of the quasicontinuum equilibrium equations with continuation. J. Sci. Comput., 37(1):19-41, 2008.

[16] M. Dobson and M. Luskin. An analysis of the effect of ghost force oscillation on quasicontinuum error. M2AN Math. Model. Numer. Anal., 43(3):591-604, 2009.

[17] M. Dobson and M. Luskin. An optimal order error analysis of the one-dimensional quasicontinuum approximation. SIAM J. Numer. Anal., 47(4):2455-2475, 2009.

[18] M. Dobson, M. Luskin, and C. Ortner. Accuracy of quasicontinuum approximations near instabilities. J. Mech. Phys. Solids, 58(10):1741-1757, 2010.

[19] M. Dobson, M. Luskin, and C. Ortner. Sharp stability estimates for the force-based quasicontinuum approximation of homogeneous tensile deformation. Multiscale Model. Simul., 8(3):782-802, 2010.

[20] M. Dobson, M. Luskin, and C. Ortner. Stability, instability, and error of the force-based quasicontinuum approximation. Arch. Ration. Mech. Anal., 197(1):179-202, 2010.

[21] W. E and J. Lu. Electronic structure of smoothly deformed crystals: Cauchy-Born rule for the nonlinear tight-binding model. Comm. Pure Appl. Math., 63(11):1432-1468, 2010.

[22] W. E and J. Lu. The electronic structure of smoothly deformed crystals: Wannier functions and the Cauchy-Born rule. Arch. Ration. Mech. Anal., 199(2):407-433, 2011.

[23] W. E and P. Ming. Analysis of the local quasicontinuum method. In Frontiers and prospects of contemporary applied mathematics, volume 6 of Ser. Contemp. Appl. Math. CAM, pages 18-32. Higher Ed. Press, Beijing, 2005.

[24] W. E and P. Ming. Cauchy-Born rule and the stability of crystalline solids: static problems. Arch. Ration. Mech. Anal., 183(2):241-297, 2007.

[25] J. L. Ericksen. The Cauchy and Born hypotheses for crystals. In Phase transformations and material instabilities in solids (Madison, Wis., 1983), volume 52 of Publ. Math. Res. Center Univ. Wisconsin, pages 61-77. Academic Press, Orlando, FL, 1984.

[26] G. Friesecke and F. Theil. Validity and failure of the Cauchy-Born hypothesis in a two-dimensional mass-spring lattice. $J$. Nonlinear Sci., 12(5):445-478, 2002.

[27] F. Legoll. Multiscale methods coupling atomistic and continuum mechanics: some examples of mathematical analysis. In Analytical and numerical aspects of partial differential equations, pages 193-245. Walter de Gruyter, Berlin, 2009.

[28] P. Lin and A. V. Shapeev. Energy-based ghost force removing techniques for the quasicontinuum method. Technical report, arXiv:0909.5437, 2010.

[29] G. Lu and E. Kaxiras. Overview of multiscale simulations of materials. In M. Rieth and W. Schommers, editors, Handbook of Theoretical and Computational Nanothechnology, volume X, pages 1-33. American Scientific Publishers, 2005.

[30] M. Luskin and C. Ortner. An analysis of node-based cluster summation rules in the quasicontinuum method. SIAM J. Numer. Anal., 47(4):3070-3086, 2009.

[31] C. Makridakis, C. Ortner, and E. Süli. A priori error analysis of two force-based atomistic/continuum models of a periodic chain. Numer. Math., 119:83-121, 2011. 
[32] C. Mora-Corral. Continuum limits of atomistic energies allowing smooth and sharp interfaces in 1D elasticity. Interfaces Free Bound., 11(3):421-446, 2009.

[33] C. Ortner. A priori and a posteriori analysis of the quasinonlocal quasicontinuum method in 1D. Math. Comp., 80(275):12651285, 2011.

[34] C. Ortner. The role of the patch test in 2d atomistic-to-continuum coupling methods. Technical report, arXiv:1101.5256, 2011.

[35] C. Ortner and A. V. Shapeev. Analysis of an energy-based atomistic/continuum coupling approximation of a vacancy in the 2d triangular lattice. Technical report, arXiv:1104.0311, 2011.

[36] C. Ortner and E. Süli. Analysis of a quasicontinuum method in one dimension. M2AN Math. Model. Numer. Anal., 42(1):5791, 2008.

[37] P. Seleson and M. Gunzburger. Bridging methods for atomistic-to-continuum coupling and their implementation. Commun. Comput. Phys., 7(4):831-876, 2010.

[38] A. V. Shapeev. Consistent energy-based atomistic/continuum coupling for two-body potentials in $1 \mathrm{~d}$ and $2 \mathrm{~d}$. Technical report, arXiv:1010.0512, 2010.

[39] V. B. Shenoy, R. Miller, E. B. Tadmor, D. Rodney, R. Phillips, and M. Ortiz. An adaptive finite element approach to atomicscale mechanics - the quasicontinuum method. J. Mech. Phys. Solids, 47(3):611-642, 1999.

[40] E. Süli, B. Jovanović, and L. Ivanović. Finite difference approximations of generalized solutions. Mathematics of Computation, 45:319-327, 1985.

[41] B. Van Koten, X. H. Li, M. Luskin, and C. Ortner. A computational and theoretical investigation of the accuracy of quasicontinuum methods. Technical report, arXiv:1012.6031, 2011.

[42] B. Van Koten and M. Luskin. Analysis of energy-based blended quasicontinuum approximations. Technical report, arXiv:1008.2138, 2011.

[43] M. Xu, R. Gracie, and T. Belytschko. A continuum-to-atomistic bridging domain method for composite lattices. Internat. J. Numer. Methods Engrg., 81(13):1635-1658, 2010.

[44] J. A. Zimmerman, R. E. Jones, and J. A. Templeton. A material frame approach for evaluating continuum variables in atomistic simulations. J. Comput. Phys., 229(6):2364-2389, 2010.

\section{Charalambos Makridakis}

Department of Applied Mathematics, University of Crete, 71409 Heraklion-Crete, Greece; and Institute of Applied and Computational Mathematics, FORTH, 71110 Heraklion-Crete, Greece

makr@tem.uoc.gr

and

Endre Süli

Mathematical Institute, University of Oxford, 24-29 St Giles', Oxford OX1 3LB, UK

suli@maths.ox.ac.uk 